\title{
The Electron-Phonon Interaction in the Presence of Strong Correlations
}

\author{
M. Grilli and C. Castellani \\ Dipartimento di Fisica, Universitá La Sapienza, Piazzale Aldo Moro, 00185 Roma, Italy
}

\begin{abstract}
We investigate the effect of strong electron-electron repulsion on the electronphonon interaction from a Fermi-liquid point of view. In particular we show that the strong interaction is responsible for vertex corrections, which are strongly dependent on the $v_{F} q / \omega$ ratio, where $v_{F}$ is the Fermi velocity and $q$ and $\omega$ are the transferred momentum and frequency respectively. These corrections generically lead to a strong suppression of the effective coupling between quasiparticles mediated by a single phonon exchange in the $v_{F} q / \omega \gg$ 1 limit. However, such effect is not present when $v_{F} q / \omega \ll 1$. Analyzing the Landau stability criterion, which involves the effective interactions in the dynamical limit, we show that a sizable electron-phonon interaction can push the system towards a phase separation instability.

A detailed analysis is then carried out using a slave-boson approach for the infinite- $\mathrm{U}$ three-band Hubbard model describing the basic structure of a $\mathrm{CuO}_{2}$ plane in copper oxides. In the presence of a coupling between the local hole density and a dispersionless optical phonon, we explicitly confirm the strong dependence of the hole-phonon coupling on the transferred momentum versus frequency ratio. We also find that the exchange of phonons leads to an unstable phase with negative compressibility already at small values of the bare hole-phonon coupling. Close to the unstable region, we detect Cooper instabilities both in s- and d-wave channels supporting a possible connection between phase separation and superconductivity in strongly correlated systems.
\end{abstract}

PACS:71.27.+a, 63.20.Kr, 74.72.-h, 74.25.Kc

\section{INTRODUCTION}

The problem of the interplay between the electron-phonon (e-ph) coupling and the (strong) electron-electron (e-e) interaction is an interesting problem, which still lacks a complete understanding. At present this topic is very hot since various facts indicate that the lattice can play a non negligible role in both the superconducting copper oxides and the fullerenes. As far as these latter materials are concerned, the strength of the interaction is still a matter of a debate, but there is a rather large agreement on the prominent role of the lattice. On the other hand, as far as copper oxides are concerned, it is generally recognized that the e-e interaction is very large in these systems, but it is the relevance of 
the lattice that is questioned. However various groups [1]- [3] claim that there are both experimental evidences and theoretical arguments supporting a prominent role of the lattice in the cuprates. Moreover recent optical experiments in the mid-infrared frequency region indicate the presence of polaronic effects [1] for the very lightly doped compounds, which are known to be strongly correlated systems.

Various issues can be addressed in investigating the role of the lattice in the presence of a strong e-e interaction. In particular two questions can be raised, which are relevant, both on a general ground as well as in the framework of high temperature superconductivity. The first question concerns the possibility of having a large phonon-mediated effective e-e coupling due to the strong mass enhancement $\left(m^{*} / m \gg 1\right)$ occurring in a strongly correlated Fermi liquid: after all the dimensionless coupling $\lambda=\gamma^{2} \nu_{0}$ ( $\gamma$ is the usual bare electron-phonon coupling and $\nu_{0}$ is the free-electron density of states) [5] could grow very large because of the density of states renormalization arising from the mass enhancement $\lambda \rightarrow \lambda=\gamma^{2} \nu^{*}$, with $\nu^{*}=\left(m^{*} / m\right) \nu_{0}$. This would have crucial consequences both on transport properties as well as on the Cooper pair formation. Moreover, a large $\lambda$ would favor the formation of polarons.

The second question regards the possible occurrence of instabilities in the electronic gas. Previous studies of single- and multi-band Hubbard models in the strong-coupling $(U \gg t)$ limit have revealed a strong tendency of these systems to undergo Phase Separation (PS) and Charge-Density-Wave (CDW) instabilities as soon as short range interactions are introduced. This occurs irrespectively to the magnetic (e.g. nearest neighbor Heisenberg coupling) [6][12] or the Coulombic (e.g. nearest neighbor repulsion) [13]- 15] nature of the short-range interaction. In the framework of interest here one can ask whether also an e-ph coupling can destabilize the electron gas. The analysis of the stability with respect to PS of a given model is particularly relevant since the investigation carried out in models displaying PS also showed that superconductivity takes place close to the instability region [8], [9], [12][14] (the Cooper pairing occurring as a precursor of PS due to the attraction eventually driving the system to the PS instability).

We anticipate here the answers to the two above questions, which will be analyzed in detail in the rest of the paper.

As far as the first problem is concerned, a general analysis performed within a standard Fermi-liquid scheme with phonons coupled with the electron density reveals a dependence of the effective phonon-mediated e-e interaction on the ratio between the transferred momentum $q$ and the frequency $\omega$, because of the vertex corrections generated by the e-e interaction. This dependence can be particularly strong in the presence of a large quasiparticle mass enhancement. As a result, when $v_{F} q / \omega \gg 1$, we find that the screening of the quasiparticles strongly enhances the vertex corrections. These corrections tend to suppress the effective interaction so that the resulting static coupling $\lambda$ is small. This is in agreement with previous calculations [17] performed in a specific model, where, however, the e-ph coupling arises from the ion-position dependence of the hopping matrix elements (the so-called covalent e-ph coupling). On the contrary, in the opposite limit $v_{F} q / \omega \ll 1$ the intraband quasiparticle screening is ineffective, the physics being dominated by high energy (e.g. interband charge-transfer $(\mathrm{CT})$ ) processes, and a large effective e-ph interaction results.

The strong dependence of the vertex corrections on the $v_{F} q / \omega$ ratio renders the analysis of the effects of the e-ph coupling particularly delicate, since different physical quantities may 
involve different $v_{F} q / \omega$ regimes. In particular the e-ph scattering in transport properties is dominated by low-energy-high-momentum processes, which suffer a strong suppression due to the large vertex corrections. As pointed out in Ref. [17], this will reduce the relevance of the e-ph scattering particularly at low doping, where the e-e interaction effects are more relevant. Similar conclusions have also been recently drawn [18] from the analysis of a single-band Hubbard model with electrons coupled with an optical dispersionless phonon.

On the other hand an affirmative answer can be given to the second question concerning the occurrence of instabilities in the electron gas. In fact, while the behaviour of $\lambda$ described above is generally true far from an instability, it will be shown that a sufficiently large e-ph coupling can instead produce a PS instability [19]. This reflects the fact that the Landau stability criterion for the symmetric Landau parameter, $F_{0}^{s}>-1$, required for a positive compressibility, involves the total (e-e and e-ph mediated) interaction in the dynamical limit $v_{F} q / \omega \rightarrow 0$. At the point where $F_{0}^{s}=-1$ the compressibility diverges and nearby, even the total static scattering amplitude (within a RPA resummation of the phonon-mediated interaction) is large and negative. Also this analysis can be carried out on a rather general ground. However, the quantitative determination of the instability conditions, being related to the subtle interplay between various interactions, depends on the couplings involved and must rely on the treatment of a specific model. For this purpose we shall consider a three-band Hubbard model describing the holes in the $\mathrm{CuO}_{2}$ planes of the high $\mathrm{T}_{\mathrm{c}}$ copper oxides. A coupling between the local hole density and a dispersionless optical phonon will be specifically considered. To deal with the electronic correlations in the strong-coupling limit we use the standard slave-boson technique within a $1 / N$ expansion. The analysis of this model explicitly detects the presence of an instability region where the compressibility of the fermion gas diverges and then becomes negative. Near the region where the system becomes unstable, superconducting instabilities are found by averaging over the Fermi surface the interaction amplitudes in the Cooper channel.

The plan of the paper is as follows. In Section II we address the two above questions working in the general framework of the Landau Fermi-liquid theory. The three-band Hubbard model with the electrons coupled with a dispersionless optical phonon is introduced in Section III. The instabilities are investigated in Section IV. Final remarks are contained in Section V.

\section{PHONON-MEDIATED EFFECTIVE INTERACTION: A FERMI-LIQUID DISCUSSION}

One of the basic concepts of Landau Fermi-liquid theory is the idea of mass renormalization. This idea is also crucially present in the most common treatments of the interacting Fermi systems. In particular it is naturally introduced in the Gutzwiller treatment of the Hubbard model [21] and is at the basis of the Mott-Hubbard transition in this model [22]. This very same basic concept recurs in other popular techniques like, e.g. the slave-boson technique [29]- 32].

In the presence of a strong interaction the mass renormalization can be very large, $m^{*} / m \gg 1$, and results in an enhancement of the quasiparticle density of states $\nu^{*}=$ $\left(m^{*} / m\right) \nu_{0}$. Then, as already discussed in the previous Section, the natural question on the consequences of this renormalization on the effective phonon mediated e-e interaction is 
whether the bare (free electron) effective e-e coupling $\lambda=\gamma^{2} \nu_{0}$ grows into $\lambda=\gamma^{2} \nu^{*}$ as an effect of the mass enhancement.

Without a significant loss of generality we address the above question discussing the case of an optical phonon coupled to the local density of electrons by a constant coupling $\gamma$ [24]. In the presence of a (possibly large) e-e interaction one has to worry about the e-ph vertex corrections involving the e-e interaction, for which no Migdal theorem can be applied. The problem is conveniently cast in the language of the standard Fermi-liquid theory [23, by using the two relations connecting the density vertex $\Lambda(q, \omega)$ and the wavefunction renormalization $z_{w}$ in the dynamic and static limits

$$
\begin{aligned}
& z_{w} \Lambda(q=0, \omega \rightarrow 0)=1 \\
& z_{w} \Lambda(q \rightarrow 0, \omega=0)=\frac{1}{1+F_{0}^{s}} .
\end{aligned}
$$

$F_{0}^{s}=2 \nu^{*} \Gamma_{\omega}$ is the standard Landau parameter and $\Gamma_{\omega}$ is the dynamic $(q=0, \omega \rightarrow 0)$ effective e-e scattering amplitude between the quasiparticles.

We first consider the effective e-e interaction arising from a single phonon exchange at lowest order in $\gamma^{2}$. Then the vertex corrections can not include phonon processes and the dynamic Landau scattering amplitude in Eq.(2) is due to the e-e interaction only. To explicitly keep memory of this limitation we append a suffix " $e$ " to $\Gamma_{\omega}$ and to any quantity not involving phononic processes. Thus $F_{0}^{s(e)}=2 \nu^{*} \Gamma_{\omega}^{e}$. The relations (1) and (2) are exact Ward identities, which must be satisfied irrespective of the details of the e-e interaction and show a drastic difference between the dynamic $(q=0, \omega \rightarrow 0)$ and static $(q \rightarrow 0, \omega=0)$ limits. Whenever the exchange of a phonon takes place, the vertex corrections must be included leading to a different behaviour of the effective interaction in the two limits. The effective dimensionless e-e interaction mediated by a single phonon exchange reads

$$
\nu^{*} \Gamma_{\mathrm{eff}}^{p h}(q, \omega)=\nu^{*} \gamma^{2} z_{w}^{e 2} \Lambda^{e 2}(q, \omega) \frac{\omega^{2}(q)}{\omega^{2}-\omega^{2}(q)}
$$

where $\omega(q)$ is the phonon dispersion. Here the presence of $z_{w}^{e}$ indicates that we are considering the effective interaction between quasiparticles and $\Lambda$ expresses the difference of the phonon coupling to the quasiparticles with respect to particles [23].

The effects of the strong $\omega-q$ dependence of the electronic density vertex $\Lambda$, in $\Gamma_{\text {eff }}(q, \omega)$ (see Eq. (3)) can be made apparent in the small $q$ and $\omega$ limits, where the relations (1) and (包) can be used. Then one obtains

$$
\begin{aligned}
\nu^{*} \Gamma_{\mathrm{eff}}^{p h}(q \rightarrow 0, \omega \rightarrow 0) & =-\gamma^{2} \nu^{*}, \quad \frac{v_{F} q}{\omega} \rightarrow 0 \\
\nu^{*} \Gamma_{\mathrm{eff}}^{p h}(q \rightarrow 0, \omega \rightarrow 0) & =-\gamma^{2} \frac{\nu^{*}}{\left(1+F_{0}^{s}\right)^{2}}= \\
& =-\gamma^{2} \frac{\kappa^{e}}{\left(1+F_{0}^{s(e)}\right)} \\
& =-\gamma^{2} \frac{\kappa^{e 2}}{\nu^{*}} ; \frac{\omega}{v_{F} q} \rightarrow 0
\end{aligned}
$$


where $\kappa^{e}=\frac{\nu^{*}}{\left(1+F_{0}^{s(e)}\right)}$ is the compressibility of the Fermi liquid in the absence of coupling with the lattice. It should be noted that, in the present case of an optical phonon, the phonon propagator tends to -1 in both limits, Eqs.(4) and (5).

The difference between the dynamic and static case can be dramatic in the case of a Fermi liquid with a large mass enhancement $m^{*} / m \gg 1$, but with a negligible compressibility renormalization $\left(\kappa^{e} \simeq \nu_{0}\right)$ [25]. In fact, in the case under consideration, $F_{0}^{s(e)}$ is proportional to the quasiparticle density of states, $\nu^{*}=\left(m^{*} / m\right) \nu_{0} \gg \nu_{0}$, and one has $F_{0}^{s(e)} \gg 1$. Then Eqs.(4) and (5) read

$$
\begin{aligned}
\nu^{*} \Gamma_{\mathrm{eff}}^{p h}(q=0, \omega \rightarrow 0) & =-\gamma^{2}\left(\frac{m^{*}}{m}\right) \nu_{0} \\
\nu^{*} \Gamma_{\mathrm{eff}}^{p h}(q \rightarrow 0, \omega=0) & =-\gamma^{2} \frac{\kappa^{e 2}}{\nu^{*}} \\
& \simeq-\gamma^{2}\left(\frac{m}{m^{*}}\right) \nu_{0}
\end{aligned}
$$

so that the effective one-phonon-mediated e-e interaction is large $\left(\sim m^{*} / m\right)$ in the dynamic limit and small $\left(\sim m / m^{*}\right)$ in the static one.

The strong $\omega-q$ dependence in Eqs.(6) and (7) concerns the small- $q$ and small- $\omega$ limits. This result relies on quite general arguments, whereas the case of finite $q$ 's and $\omega$ 's will need the analysis of a specific model and will be discussed in the context of the three-band Hubbard model in the next sections. Our expectation, which will be confirmed by the analysis in Section IV, is that the product $z_{w} \Lambda$ will be roughly of order one (dynamical limit) all over the region outside the particle-hole continuum, while it will strongly deviate from unity in the region of the particle-hole continuum, where screening processes take place. As a consequence the e-ph coupling (and the e-e interaction mediated by phonons) will be depressed by the e-e interaction in all processes involving small energy and large momenta (as in the low energy lifetime and transport).

The above conclusion on the irrelevance of the e-ph coupling is based on a lower order analysis in $\gamma^{2}$. It evidently contrasts with the fact that the Landau stability criterion $F_{0}^{s}>-1$ involves the full e-e interaction in the dynamical limit, where the e-ph coupling is not depressed by the "pure" e-e vertex corrections. Within the same limits discussed above (lowest order in $\gamma^{2}$ ) we have

$$
F_{0}^{s}=\nu^{*}\left(\Gamma_{\omega}^{e}-\gamma^{2}\right)
$$

indicating that a sizable $\gamma^{2}$ can indeed lead to $F_{0}^{s}<-1$. (Note that $m^{*} / m \gg 1$ requires a large bare repulsion in units of the bare Fermi energy, as in the single- or multi-band Hubbard model near the metal-insulator transition, but this does not imply a large $\left.\Gamma_{\omega}^{e}\right)$. The lowest order analysis showing the depression of the e-ph coupling in the low energy processes maintains its full validity with respect to the inclusion of higher order terms provided $F_{0}^{s}$ in Eq.(8) is still of order $m^{*} / m$. On the contrary, near the instability condition $F_{0}^{s}=-1$, the phonon contributions to the vertex cannot be neglected and the e-ph interaction is relevant even in the static limit. To clarify this point we extend our analysis considering a bubble resummation of the one-phonon processes, in the framework of a standard RPA approach for the quasiparticles. 
As a first step we introduce an effective dynamic e-e scattering amplitude $\Gamma_{\omega}^{e}(q, \omega)$ with no phonon processes included in it. Here and below, the subscript $\omega$ indicates that no intraband screening processes are present in the considered quantity. The $q \rightarrow 0$ and $\omega \rightarrow 0$ limit of $\Gamma_{\omega}^{e}(q, \omega)$ reproduces the above Landau dynamic scattering amplitude $\Gamma_{\omega}^{e}$ of the Fermi liquid in the absence of e-ph coupling.

The second ingredient of our analysis is the intraband Lindhardt polarization bubble

$$
\Pi(q, \omega)=2 \sum_{k} \frac{f\left(E_{k+\frac{q}{2}}\right)-f\left(E_{k-\frac{q}{2}}\right)}{E_{k+\frac{q}{2}}-E_{k-\frac{q}{2}}-\omega}
$$

where $f\left(E_{k}\right)$ is the Fermi function and $E_{k}$ is the quasiparticle band. Here we have included the spin multiplicity in the definition of $\Pi(q, \omega)$. This bubble can be dressed with all possible intraband and interband purely electronic screening processes within a RPA resummation leading to

$$
\widetilde{\Pi}^{e}(q, \omega)=\frac{\Pi(q, \omega)}{1+\Gamma_{\omega}^{e}(q, \omega) \Pi(q, \omega)}
$$

Finally we consider the self-energy corrections to the phonon propagator arising from the purely electronic processes. Once both the interband and the intraband screening of the quasiparticles is taken into account one obtains a phonon propagator with a self-energy correction given by $g^{2} \widetilde{\Pi}$

$$
\begin{aligned}
\mathcal{D}(q, \omega) & =\frac{\omega^{2}(q)}{\omega^{2}-\omega^{2}(q)\left(1-\gamma^{2} \widetilde{\Pi}^{e}(q, \omega)\right)} \\
& =\frac{\omega^{2}(q)}{\omega^{2}-\omega^{2}(q) \frac{1+\widetilde{\Gamma}_{\omega}(q, \omega) \Pi(q, \omega)}{1+\Gamma_{\omega}^{e}(q, \omega) \Pi(q, \omega)}} .
\end{aligned}
$$

Notice that the quantity

$$
\widetilde{\Gamma}_{\omega}(q, \omega) \equiv \Gamma_{\omega}^{e}(q, \omega)+\Gamma_{\omega}^{p h}(q=0, \omega \rightarrow 0)=\Gamma_{\omega}^{e}(q, \omega)-\gamma^{2}
$$

has been introduced. Taking the limit $q \rightarrow 0$ first and then $\omega \rightarrow 0, \widetilde{\Gamma}$ becomes the Landau dynamic scattering amplitude in the presence of an e-ph coupling.

We now can evaluate the total scattering amplitude splitting the contribution of the purely electronic processes and the contribution of the processes also involving phonons

$$
\nu^{*} \Gamma(q, \omega)=\frac{\nu^{*} \Gamma_{\omega}^{e}(q, \omega)}{1+\Gamma_{\omega}^{e}(q, \omega) \Pi(q, \omega)}+\frac{1}{\left[1+\Gamma_{\omega}^{e}(q, \omega) \Pi(q, \omega)\right]^{2}} \frac{\nu^{*} \omega^{2}(q) \gamma^{2}}{\omega^{2}-\omega^{2}(q) \frac{1+\widetilde{\Gamma}_{\omega}(q, \omega) \Pi(q, \omega)}{1+\Gamma_{\omega}^{e}(q, \omega) \Pi(q, \omega)}}
$$

It is important to notice that vertex corrections have been included in the second term by specializing Eqs.(四) and (2) into $z_{w}^{e} \Lambda^{e}=\left[1+\Gamma_{\omega}^{e}(q, \omega) \Pi(q, \omega)\right]^{-1}$. Nevertheless, now the attraction mediated by this second term can be large even in the static limit if the denominator of the phonon propagator becomes small. In fact, in the $\omega \rightarrow 0$ limit one has for the attractive part of Eq. (13) 


$$
-\frac{1}{\left[1+\Gamma_{\omega}^{e}(q, \omega) \Pi(q, \omega)\right]} \frac{\nu^{*} \gamma^{2}}{1+\widetilde{\Gamma}_{\omega}(q, 0) \Pi(q, 0)} .
$$

From this expression one can see that the condition for a diverging static scattering amplitude, eventually leading to an instability of the Fermi liquid, results in the condition on the Landau dynamic scattering amplitude

$$
1+\widetilde{\Gamma}_{\omega}(q \rightarrow 0, \omega=0) \Pi(q \rightarrow 0, \omega=0)=0
$$

equivalent to the usual $1+F_{0}^{s}=0$, Eq.(8), since in this limit $\Pi(q, \omega)$ reduces to $2 \nu^{*}$.

This result shows that a Fermi liquid can indeed be destabilized by the coupling of the quasiparticles to the lattice even in the presence of a very strong e-e repulsion. It must be noticed that this instability does not imply the full phonon softening, since the determination of the renormalized phonon frequency $\widetilde{\omega}(q)$ requires the value of

$$
\frac{1+\widetilde{\Gamma}_{\omega}(q, \omega) \Pi(q, \omega)}{1+\Gamma_{\omega}^{e}(q, \omega) \Pi(q, \omega)}
$$

in a range of frequencies, where the numerator does not vanish. The instability appears instead as an overdamping of the zero sound driven by the e-ph mediated attraction.

The quantitative determination of the needed strength of the e-ph coupling in order to have an instability must rely on the analysis of a specific model. In fact, as it is apparent in its definition, Eq. (12), the strength and the sign of $\widetilde{\Gamma}_{\omega}$ depend on the balance between $\Gamma_{\omega}^{e}(q, \omega)$ and $\gamma^{2}$. Moreover, as we shall explicitly see in the model treated in the following sections, $\Gamma_{\omega}^{e}(q, \omega)$ in turn results from a cancellation between the strong bare repulsion and the strong interband screening. How much is left from this cancellation depends on the specific model one is dealing with.

\section{THE MODEL}

The model we consider is represented by the following Hamiltonian

$$
\begin{aligned}
H & =\varepsilon_{d}^{0} \sum_{i \sigma} d_{i \sigma}^{\dagger} d_{i \sigma}+\varepsilon_{p}^{0} \sum_{i \sigma}\left(p_{i \sigma x}^{\dagger} p_{i \sigma x}+p_{i \sigma y}^{\dagger} p_{i \sigma y}\right)+\sum_{<i j>\sigma}\left(t_{i j} d_{i \sigma}^{\dagger} p_{j \sigma x}+x \rightarrow y+\text { h.c. }\right) \\
& +\sum_{<i j>\sigma}\left(t_{p p i j} p_{i \sigma x}^{\dagger} p_{j \sigma y}+\text { h.c. }\right)+U_{d} \sum_{i} n_{d i \uparrow} n_{d i \downarrow}+\omega_{0} \sum_{i} A_{i}^{\dagger} A_{i} \\
& -\sum_{i, \sigma}\left(A_{i}+A_{i}^{\dagger}\right)\left[G_{d}\left(n_{d i}-\left\langle n_{d i}\right\rangle\right)+G_{p}\left(n_{p i}-\left\langle n_{p i}\right\rangle\right)\right] .
\end{aligned}
$$

where $\varepsilon_{d}^{0}$ and $\varepsilon_{p}^{0}$ are the $\mathrm{Cu}$ and $\mathrm{O}$ energy levels respectively, $t_{i j}= \pm t_{p d}$ is the $\mathrm{Cu}$-O hybridization, $t_{p p i j}= \pm t_{p p}$ is the nearest neighbor O-O hybridization (for the choice of the orbital phases and the related choice of the sign of the hopping constants, see, e.g., Ref. [26]). $U_{d}$ is the on site repulsion between holes on copper sites. Starting from a $\mathrm{Cu}\left(3 d^{10}\right)-\mathrm{O}\left(2 p^{6}\right)$ vacuum state, holes on copper $d_{x^{2}-y^{2}}$ orbitals or on oxygen $p_{x}$ or $p_{y}$ orbitals at site $i$ are created by the $d_{i}^{\dagger}$ and $p_{i}^{\dagger}$ operators respectively. $n_{d i}=\sum_{\sigma} d_{i \sigma}^{\dagger} d_{i \sigma}$ is the total density per cell of holes on copper, while $n_{p i}=\sum_{\sigma, \alpha=x, y} p_{i, \sigma, \alpha}^{\dagger} p_{i, \sigma, \alpha}$ is the total density per cell of holes on oxygen. The 
boson creation operator $A_{i}^{\dagger}$ creates a dispersionless phonon with frequency $\omega_{0}$ coupled to the local density of copper and oxygen holes by the coupling constants $G_{d}$ and $G_{p}$ respectively [27]. These couplings can arise from the dependence of the interionic Coulombic repulsion on the relative position as well as on the hole occupation. An estimate can be obtained when only the nearest neighbor Coulomb interaction $V\left(\left|r_{i}-r_{j}\right|\right) n_{d i} n_{p j}$ is considered. Then, a first order expansion on the ion displacement and on the charge fluctuations leads to an e-ph coupling of the form appearing in the Hamiltonian (17), with

$$
\left.G_{d} \propto \frac{\partial V}{\partial r_{i}}\right|_{R}\left\langle n_{p j}\right\rangle ;\left.\quad G_{p} \propto \frac{\partial V}{\partial r_{j}}\right|_{R}\left\langle n_{d i}\right\rangle
$$

where $R$ is the equilibrium distance between nn ions. Therefore, two different constants, $G_{d}$ and $G_{p}$, have been introduced owing to the different average occupations of the $d$ and $p$ orbitals and to the difference in ionic masses entering the standard normalization factors implicitly included in the definition of the $G$ 's. However, we do not want to stress very much in the present context the relevance of the specific form of the e-ph coupling appearing in Eq.(18) [28]. In fact we do not address the specific aspects (like, e.g., symmetries and strengths) of the phonons in the cuprates, our issue being the understanding of the general properties of an Holstein [16] phonon in a strongly correlated system.

A similar three-band Hubbard model was considered in Ref. [17], where, however, an intersite "covalent" e-ph coupling was considered arising from the ion-position dependence of the hopping integrals.

Since our investigation concerns the interplay between strong interactions and the phonons we take the $U_{d} \rightarrow \infty$ limit. In a standard way we handle the no-double-occupancy constraint on copper sites by means of the slave-boson technique [29]- [32]. Therefore, after performing the usual substitution $d_{i \sigma}^{\dagger} \rightarrow d_{i \sigma}^{\dagger} b_{i}, \quad d_{i \sigma} \rightarrow b_{i}^{\dagger} d_{i \sigma}$ the constraint becomes $\sum_{\sigma} d_{i \sigma}^{\dagger} d_{i \sigma}+b_{i}^{\dagger} b_{i}=1$. To equip the model with a formal small expansion parameter, we introduce a standard large-N expansion [30], where the spin index $\sigma$ runs from 1 to $N$. The constraint is relaxed to assume the form $\sum_{\sigma} d_{i \sigma}^{\dagger} d_{i \sigma}+b_{i}^{\dagger} b_{i}=\frac{N}{2}$ and the suitable rescaling of the hopping $t_{p d} \rightarrow t_{p d} / \sqrt{N}$ must, in this model, be joined by the similar rescaling of the hole-phonon coupling $G \rightarrow G / \sqrt{N}$ in order to compensate for the presence of $N$ fermionic degrees of freedom. Once these transformations are carried out the partition function of the final model can be written as a functional integral

$$
\begin{aligned}
Z & =\int D p_{\alpha \sigma}^{\dagger} D p_{\alpha \sigma} D d_{\sigma}^{\dagger} D d_{\sigma} D b^{\dagger} D b D \lambda D A D A^{\dagger} e^{-\int_{0}^{\beta} S d \tau}, \\
S & =\sum_{i}\left[\sum_{\sigma} d_{i \sigma}^{\dagger} \frac{\partial d_{i \sigma}}{\partial \tau}+\sum_{\sigma \alpha=x, y} p_{i \sigma \alpha}^{\dagger} \frac{\partial p_{i \sigma \alpha}}{\partial \tau}+b_{i}^{\dagger} \frac{\partial b_{i}}{\partial \tau}+A_{i}^{\dagger} \frac{\partial A_{i}}{\partial \tau}\right]+\sum_{i}\left[i \lambda_{i}\left(b_{i}^{\dagger} b_{i}-\frac{N}{2}\right)\right]+H \\
H & =\sum_{i, \sigma} d_{i \sigma}^{\dagger} d_{i \sigma}\left(\varepsilon_{d}^{0}+i \lambda_{i}\right)+\varepsilon_{p}^{0} \sum_{i, \sigma, \alpha=x, y} p_{i, \sigma, \alpha}^{\dagger} p_{i, \sigma, \alpha} \\
& -\frac{t_{p d}}{\sqrt{N}} \sum_{i, \sigma}\left[\left(p_{i, \sigma, x}^{\dagger}-p_{i, \sigma,-x}^{\dagger}+p_{i, \sigma, y}^{\dagger}-p_{i, \sigma,-y}^{\dagger}\right) d_{i \sigma} b_{i}^{\dagger}+c . c .\right] \\
& -t_{p p} \sum_{i \sigma}\left[p_{i+x, \sigma, x}^{\dagger}\left(p_{i, \sigma,-y}-p_{i, \sigma, y}+p_{i+2 x, \sigma, y}-p_{i+2 x, \sigma,-y}\right)+c . c .\right] \\
& -\frac{1}{\sqrt{N}} \sum_{i, \sigma}\left(A_{i}+A_{i}^{\dagger}\right)\left[G_{d}\left(n_{d i}-\left\langle n_{d i}\right\rangle\right)+G_{p}\left(n_{p i}-\left\langle n_{p i}\right\rangle\right)\right]+\omega_{0} \sum_{i} A_{i}^{\dagger} A_{i} .
\end{aligned}
$$


where a local Lagrange multiplier field $\lambda$ has been introduced to implement the local constraint forbidding the double occupancy on copper.

At the mean-field $(N=\infty)$ level, the model of Eqs.(19)-(21) is equivalent to the standard, purely electronic three-band Hubbard model without coupling to the phonons, which has been widely considered in the literature [33], [26]. In fact, at mean field level no role is played by the phonons because our electron-lattice coupling depends on the difference between the local and the average density and this difference naturally vanishes in the mean-field approximation [34]. The model displays a $\mathrm{T}=0$ Fermi-liquid behaviour for any finite doping $\delta$, where $\delta$ is the deviation from half-filling, when one hole per cell is present in the system. In the Fermi-liquid case the mean-field value of the slave-boson field $b_{0}$ multiplicatively renormalizes the hopping thus enhancing the effective mass of the quasiparticles $\left(b_{0} / \sqrt{N} \leq\right.$ $\sqrt{1 / 2}$ ). Moreover, at this level the single particle self-energy does not introduce a finite quasiparticle lifetime. Then, in this model the single particle Green function of the physical fermions at $N=2$ has a quasiparticle pole with a finite residue given by the square of the mean-field value of the slave-boson field $b_{0}^{2}$.

On the other hand, at half-filling the system becomes an insulator if the bare chargetransfer energy difference $\varepsilon_{p}^{0}-\varepsilon_{d}^{0}-4 t_{p p}$ is larger than a critical value ranging from $3.35 t_{p d}$ when $t_{p p}=0$ [33] to a smaller value $\approx 2.5 t_{p d}$ when $t_{p p}=0.5 t_{p d}$ [26]. In the insulating phase $b_{0}$ vanishes leading to an infinite quasiparticle mass $\left(m^{*} / m=\infty\right)$ and to a vanishing quasiparticle spectral weight [35].

In order to get new physical effects from the presence of the coupling with the phonons, one needs to consider the fluctuations of the bosonic fields. Since only a particular combination $a=\left(A^{\dagger}+A\right) /(2 \sqrt{N})$ of the phonon fields $A$ and $A^{\dagger}$ is coupled to the fermions, it is convenient to use the field $a$ and to integrate out the orthogonal combination $\widetilde{a}=\left(A-A^{\dagger}\right) /(2 \sqrt{N})$. Then the quadratic action for the boson field $a$ reads

$$
H_{\text {phon }}=N \sum_{n, i} \frac{\omega_{n}^{2}+\omega_{0}^{2}}{\omega_{0}} a_{i}^{\dagger} a_{i},
$$

where we have transformed the imaginary time into Matsubara frequencies. Moreover, working in the radial gauge [31], the phase of the field $b_{i}=\sqrt{N} r_{i} \exp (-i \phi)$ is gauged away and only the modulus field $r_{i}$ is kept, while $\lambda_{i}$ acquires a time dependence $\lambda_{i} \rightarrow$ $\lambda_{i}+\partial_{\tau} \phi_{i}$. Thus one can define a three-component field $A^{\mu}=(\delta r, \delta \lambda, a)$ where the timeand space-dependent components are the fluctuating part of the boson fields $r_{i}=r_{0}\left(1+\delta r_{i}\right)$, $\lambda_{i}=-i \lambda_{0}+\delta \lambda_{i}$ and $a_{i}$. Writing the Hamiltonian of coupled fermions and bosons as $H=$ $H_{M F}+H_{\text {bos }}+H_{\text {int }}$, where $H_{M F}$ is the mean-field Hamiltonian quadratic in the fermionic fields, $H_{\text {bos }}$ is the purely bosonic part, also including the terms with the $a, r$ and $\lambda$ bosons appearing in the action (20) and in $H_{\text {phon }}$, Eq.(22). $H_{\text {int }}$ contains the fermion-boson interaction terms. More explicitly, Fourier transforming to the momentum space, the bosonic part reads

$$
H_{\mathrm{bos}}=N \sum_{q \mu \nu} A^{\mu}(q) B^{\mu \nu}(q) A^{\nu}(-q)
$$

without explicitly indicating the frequency dependence for the sake of simplicity. The form of Eqs.(20)-(22) allows to determine the matrix $B^{\mu, \nu}$, whose elements are all zero except for $B^{1,1}=r_{0}^{2} \lambda_{0}, B^{1,2}=B^{2,1}=i r_{0}^{2}, B^{3,3}=\left(\omega_{n}^{2}+\omega_{0}^{2}\right) / \omega_{0}$. 
To simplify the notation in $H_{M F}$ and in $H_{\text {int }}$, we introduce a three-component fermionic field $\Psi_{k \sigma \alpha} \equiv\left(d_{k \sigma}, i p_{x k \sigma}, i p_{y k \sigma}\right)$ describing in momentum space the fermions in the orbital basis. Then $H_{M F}$ can compactly be written as $H_{M F}=\sum_{k \sigma \alpha \beta} H_{M F}^{\alpha \beta}(k) \Psi_{k \sigma \alpha}^{\dagger} \Psi_{k \sigma \beta}$, where

$$
H_{M F}(k)=\left(\begin{array}{ccc}
\varepsilon_{d} & -2 r_{0} t_{p d} \sin \left(k_{x} / 2\right) & -2 r_{0} t_{p d} \sin \left(k_{y} / 2\right) \\
-2 r_{0} t_{p d} \sin \left(k_{x} / 2\right) & \varepsilon_{p} & -2 t_{p p} \beta_{k} \\
-2 r_{0} t_{p d} \sin \left(k_{y} / 2\right) & -2 t_{p p} \beta_{k} & \varepsilon_{p}
\end{array}\right)
$$

with $\beta_{k} \equiv 2 \sin \left(k_{x} / 2\right) \sin \left(k_{y} / 2\right)$ and $\varepsilon_{d}=\varepsilon_{d}^{0}+\lambda_{0}$ being the mean-field-renormalized energy level of the copper $d$ orbitals. The above matrix can be put in diagonal form by a unitary transformation to the quasiparticle basis $\tilde{\Psi}_{k \sigma \alpha}=\sum_{\beta} U_{\alpha \beta}(k) \Psi_{k \sigma \beta}$ leading to $H_{M F}=\sum_{k \sigma \alpha} E_{\alpha}(k) \tilde{\Psi}_{k \sigma \alpha}^{\dagger} \tilde{\Psi}_{k \sigma \alpha}$, where $E_{1,2,3}(k)$ are the mean-field-renormalized quasiparticle bands (we choose the band 1 as the lowest one, so that the Fermi level lays in this band for filling $\delta<1$ ). This formalism allows to write the boson-fermion interaction as

$$
\begin{aligned}
H_{\mathrm{int}} & =\sum_{k, q, \sigma} \Psi_{k+\frac{q}{2} \sigma}^{\dagger} \Lambda^{\mu}(k, q) \Psi_{k-\frac{q}{2} \sigma} A^{\mu}(q) \\
& =\sum_{k, q, \sigma} \tilde{\Psi}_{k+\frac{q}{2} \sigma}^{\dagger} \tilde{\Lambda}^{\mu}(k, q) \tilde{\Psi}_{k-\frac{q}{2} \sigma} A^{\mu}(q) .
\end{aligned}
$$

The fermion-component index has been dropped and the $(3 \times 3)$ boson-fermion interaction vertices $\Lambda^{\mu}$ in the orbital operator basis can be obtained from Eq.(21)

$$
\begin{gathered}
\Lambda^{1}=-2 r_{0} t_{p d}\left(\begin{array}{ccc}
0 & \sin \left(\frac{k_{x}-q_{x} / 2}{2}\right) & \sin \left(\frac{k_{y}-q_{y} / 2}{2}\right) \\
\sin \left(\frac{k_{x}+q_{x} / 2}{2}\right) & 0 & 0 \\
\sin \left(\frac{k_{y}+q_{y} / 2}{2}\right) & 0 & 0
\end{array}\right), \\
\Lambda^{2}=\left(\begin{array}{lll}
i & 0 & 0 \\
0 & 0 & 0 \\
0 & 0 & 0
\end{array}\right), \quad \Lambda^{3}=\left(\begin{array}{ccc}
-2 G_{d} & 0 & 0 \\
0 & -2 G_{p} \cos \frac{q_{x}}{2} & 0 \\
0 & 0 & -2 G_{p} \cos \frac{q_{y}}{2}
\end{array}\right),
\end{gathered}
$$

while the quasiparticle vertices $\tilde{\Lambda}_{\alpha \beta}^{\mu}(k, q)$ are defined as

$$
\tilde{\Lambda}^{\mu}(k, q)=U\left(k+\frac{q}{2}\right) \Lambda^{\mu}(k, q) U^{\dagger}\left(k-\frac{q}{2}\right) .
$$

Introducing the fermionic bubbles coupled to the various bosons

$$
\Pi^{\mu \nu}\left(q, \omega_{m}\right)=\sum_{k, \alpha, \beta} \frac{f\left(E_{\alpha}\left(k+\frac{q}{2}\right)\right)-f\left(E_{\beta}\left(k-\frac{q}{2}\right)\right)}{E_{\alpha}\left(k+\frac{q}{2}\right)-E_{\beta}\left(k-\frac{q}{2}\right)-i \omega_{m}} \tilde{\Lambda}_{\alpha \beta}^{\mu}(k, q) \tilde{\Lambda}_{\beta \alpha}^{\nu}(k,-q) .
$$

one can define the boson propagator

$$
\begin{aligned}
D^{\mu \nu}\left(q, \omega_{m}\right) & =<A^{\mu}\left(q, \omega_{m}\right) A^{\nu}\left(-q,-\omega_{m}\right)> \\
& =N^{-1}\left(2 B+\Pi\left(q, \omega_{m}\right)\right)_{\mu \nu}^{-1}
\end{aligned}
$$

The factor 2 multiplying the boson matrix B arises from the fact that the bosonic fields in the presently used radial gauge are real. 
The set of formal tools is then completed by the introduction of the effective scattering amplitude between the quasiparticles in the lowest band

$$
\Gamma\left(k, k^{\prime} ; q, \omega\right)=-\sum_{\mu \nu} \tilde{\Lambda}_{11}^{\mu}\left(k^{\prime},-q\right) D^{\mu \nu}(q, \omega) \tilde{\Lambda}_{11}^{\nu}(k, q) .
$$

Then the scattering amplitude in the Cooper channel is given by

$$
\Gamma^{C}\left(k, k^{\prime} ; \omega\right)=-\sum_{\mu \nu} \tilde{\Lambda}_{11}^{\mu}\left(\frac{k+k^{\prime}}{2},-k+k^{\prime}\right) D^{\mu \nu}\left(q=k-k^{\prime}, \omega\right) \tilde{\Lambda}_{11}^{\nu}\left(-\frac{k+k^{\prime}}{2}, k-k^{\prime}\right)
$$

It should be noted that the boson propagators are of order $1 / \mathrm{N}$ while the occurrence of a bare fermionic bubble leads to a spin summation and is therefore associated with a factor N. Thus, in this $1 / \mathrm{N}$ approach, the quasiparticle scattering amplitudes are residual interactions of order $1 / \mathrm{N}$. The matrix form of the static density-density correlation function at the leading order is

$$
\begin{aligned}
P_{\alpha \beta}(q, \omega=0) & =\frac{1}{N} \sum_{\sigma \sigma^{\prime}}<n_{\alpha \sigma}(q) n_{\beta \sigma^{\prime}}(-q)> \\
& =P_{\alpha \beta}^{0}(q, \omega=0)+N \sum_{\mu \nu} \chi_{\alpha \mu}^{0}(q, \omega=0) D^{\mu \nu}(q, \omega=0) \chi_{\nu \beta}^{0}(q, \omega=0)
\end{aligned}
$$

where

$$
P_{\alpha \beta}^{0}(q, \omega)=\frac{1}{N} \sum_{\sigma \sigma^{\prime}}<n_{\alpha \sigma}(q) n_{\beta \sigma^{\prime}}(-q)>_{0}
$$

is the orbital bare density-density correlation function, and

$$
\chi_{\alpha \mu}^{0}(q, \omega)=\frac{1}{N} \sum_{\sigma \sigma^{\prime}}<n_{\alpha \sigma}(q) \sum_{k ; \gamma, \delta} \Psi_{k \sigma^{\prime} \gamma}^{\dagger} \Lambda_{\gamma \delta}^{\mu}(k, q) \Psi_{k+q \sigma^{\prime} \delta}>_{0}
$$

where $\alpha=d, p_{x}, p_{y}$, and $\mu=1,2$ and 3 .

\section{THE DYNAMICAL ANALYSIS}

We now analyze the interplay between lattice and holes in the strongly correlated system represented by the model of Eqs.(19)-(21) focusing on the possible occurrence of instabilities.

Two mechanisms for driving a system to an instability can in principle be devised. A first one requires the complete softening of a collective mode leading to a ground state with different symmetries. For instance, in our specific model, this mechanism could imply the softening of the phonon leading to a structural transition. To investigate this mechanism a dynamical analysis of the collective modes of the system will be carried out in Subsection A.

However, as we generally found out in Section II, an instability can also occur when the Landau criterion for stability $F_{0}^{s}>-1$ is violated (and usually a PS will then take place). In this case, the instability shows up as an overdamping of the zero-sound mode, the other collective modes remaining massive, and is a result of the delicate unbalance between the 
repulsive and the attractive forces present in the system. Subsections B and C are devoted to this particular aspect, focusing on the role of the various screening processes in determining the instability.

The result of the analyses performed in Subsections A, B and C is that the model of Eqs.(19)-(21) undergoes an instability driven by the second of the above mechanisms, and, in general, no phonon softening is detected. A similar phenomenon [13]- 15] occurs in the three-band Hubbard model in the presence of a nearest neighbor Coulombic repulsion. In this latter case it is the attraction due to copper-oxygen CT fluctuations, which determines a PS instability without being accompanied by any collective-mode softening.

\section{A. The collective modes}

The collective modes appear as poles of the density susceptibilities. However an important remark is that the poles of the density susceptibilities $\chi$ coincide with the poles of the boson propagator $D^{\mu \nu}(q, \omega)$, which therefore contain all of the relevant informations on the collective modes. In particular, to find the dispersion of these modes $\omega=\omega(q)$, one has to solve the equation

$$
\operatorname{det}(2 B+\Pi)=0
$$

Quite generally the charge-density fluctuations in the three-band model can be decomposed into the fluctuations of a non-conserved field, $n_{d}-n_{p}$, coupled to a fluctuating conserved field, $n_{d}+n_{p}$. The mode describing the propagating total density fluctuations is the zerosound mode. This mode is massless, since in the model of Eqs.(20)-(22), no long-range interaction among the holes is included, which would otherwise transform this mode into a massive plasmon. In the presence of an overall attraction $\left(0 \geq F_{0}^{s}>-1\right)$, the zero-sound mode enters into the particle-hole continuum and becomes damped. On the other hand the p-d CT fluctuations, being described by a non-conserved field, will contribute to the CT "optical-like" mode. Of course these fluctuations are dynamically coupled to the phonon mode.

In order to simplify our analysis we first consider the $q=0$ limit because in this limit all intraband fermionic bubbles vanish thereby leaving the boson propagator in a much more treatable form. Physically this is due to the fact that in the $q=0$ limit the total density field $\rho(q)=n_{d}(q)+n_{p}(q)=\left(1 / \sqrt{N_{s}}\right) \sum_{k} \tilde{\Psi}_{k+\frac{q}{2} \sigma}^{\dagger} \tilde{\Lambda}^{+}(k, q) \tilde{\Psi}_{k-\frac{q}{2} \sigma}$ with

$$
\Lambda^{+}=\left(\begin{array}{ccc}
1 & 0 & 0 \\
0 & \cos \frac{q_{x}}{2} & 0 \\
0 & 0 & \cos \frac{q_{y}}{2}
\end{array}\right)
$$

decouples from the dynamics because of particle conservation. To allow for a simpler analytic treatment we neglect in this section the direct oxygen-oxygen overlap $\left(t_{p p}=0\right)$. Thus the boson-quasiparticle vertices simplify to the form

$$
\tilde{\Lambda}_{12}^{\mu}(k, q=0)=\frac{2 r_{0} t_{p d} \gamma_{k}}{R_{k}}\left(\begin{array}{c}
\Delta \\
i \\
2 G
\end{array}\right)
$$




$$
\tilde{\Lambda}_{13}^{\mu}(k, q=0)=\frac{2 r_{0} t_{p d} \gamma_{k}}{R_{k}}\left(\begin{array}{c}
0 \\
0 \\
2 G
\end{array}\right)
$$

where $\Delta \equiv \varepsilon_{p}^{0}-\varepsilon_{d}$ is the mean-field-renormalized atomic level difference and $G \equiv G_{p}-G_{d}$. Then the following expression for the polarization bubble results

$$
\Pi^{\mu \nu}(q=0, \omega)=-2 r_{0}^{2}\left(\begin{array}{ccc}
\Delta^{2} & i \Delta & 2 \Delta G \\
i \Delta & -1 & 2 i G \\
2 \Delta G & 2 i G & 4 G^{2}
\end{array}\right) I(\omega)
$$

where

$$
I(\omega)=\frac{1}{N_{s}} \sum_{k} \frac{4 t_{p d}^{2} \gamma_{k}^{2}}{R_{k}\left(R_{k}^{2}-\omega^{2}\right)}
$$

Using Eq.(27) one obtains the following expression for $D^{-1}$

$$
D^{-1}(q=0, \omega)=2 N r_{0}^{2}\left(\begin{array}{ccc}
\lambda_{0}-\Delta^{2} I & i(1-\Delta I) & -2 \Delta I G \\
i(1-\Delta I) & I & -2 i I G \\
-2 \Delta I G & -2 i I G & -4 I G^{2}+\frac{\omega_{0}^{2}-\omega^{2}}{r_{0}^{2} \omega_{0}}
\end{array}\right)
$$

and the determinant can be evaluated as

$$
\operatorname{det} \frac{1}{N} D^{-1}(0, \omega)=\left(2 r_{0}^{2}\right)^{3}\left[\frac{\omega_{0}^{2}-\omega^{2}}{\omega_{0}}\left(1-I\left(2 \Delta-\lambda_{0}\right)\right)-4 G^{2} I\right] .
$$

The analytical treatment can then proceed further introducing the small $r_{0}^{2}$ approximation. This is justified at low doping deep inside the region of parameters where the system is insulating at half-filling. We recall that the model we are considering at leading order in $1 / N$ has a vanishing $r_{0}^{2}$ approaching the insulating regime. Then it is convenient to rewrite $I(\omega)$ as

$$
I(\omega)=\frac{\lambda_{0}}{\Delta^{2}-\omega^{2}}\left[1-\alpha(\omega) r_{0}^{2}\right]
$$

with

$$
\alpha(\omega) \equiv \frac{1}{\lambda_{0} N_{s}} \sum_{k} \frac{64 t_{p d}^{4} \gamma_{k}^{4}}{R_{k}\left(R_{k}^{2}-\omega^{2}\right)}
$$

so that the determinant assumes the form

$$
\begin{aligned}
\operatorname{det} \frac{1}{N} D^{-1}(0, \omega) & =\frac{8 r_{0}^{4}}{\omega_{0}\left(\Delta^{2}-\omega^{2}\right)}\left[\left(\omega_{0}^{2}-\omega^{2}\right)\left[\left(\Delta-\lambda_{0}\right)^{2}-\omega^{2}\right]\right. \\
& \left.+\lambda_{0} r_{0}^{2}\left[-4 G^{2} \omega_{0}+\left(\omega_{0}^{2}-\omega^{2}\right)\left(2 \Delta-\lambda_{0}\right) \alpha(\omega)\right]\right] .
\end{aligned}
$$

By expanding $\alpha$ at zeroth order in $r_{0}^{2}$

$$
\alpha(\omega) \approx \frac{16 t_{p d}^{2} \gamma_{4}}{\gamma_{2}} \frac{1}{\Delta^{2}-\omega^{2}}
$$


with $\gamma_{2} \equiv\left(1 / N_{s}\right) \sum_{k} \gamma_{k}^{2}=1 / 2+2 / \pi^{2} \simeq 0.702$ and $\gamma_{4} \equiv\left(1 / N_{s}\right) \sum_{k} \gamma_{k}^{4}=5 / 8+4 / \pi^{2} \simeq 1.03$ one obtains the expression of the determinant at first order in $r_{0}^{2}$. It is immediate to recognize that, taking the $r_{0} \rightarrow 0$ limit, one obtains two collective modes

$$
\begin{aligned}
& \omega^{2}=\left(\Delta-\lambda_{0}\right)^{2} \equiv \omega_{e x c}^{2} \\
& \omega^{2}=\omega_{0}^{2}
\end{aligned}
$$

corresponding to the excitonic charge-transfer mode $\left(n_{p}-n_{d}\right.$ fluctuations $)$ and to the bare phonon mode. At this leading level in the $1 / \mathrm{N}$ expansion the two modes do not mix. On the other hand the equation $\operatorname{det} D^{-1}=0$ can be solved giving

$$
\begin{aligned}
\omega^{2} & =\frac{1}{2}\left[\omega_{0}^{2}+\omega_{e x c}^{2}+r_{0}^{2} C(\bar{\omega}) \lambda_{0}\right. \\
& \left. \pm\left[\left(\omega_{0}^{2}-\omega_{e x c}^{2}\right)^{2}+\lambda_{0} r_{0}^{2}\left[2 C(\bar{\omega})\left(\omega_{e x c}^{2}-\omega_{0}^{2}\right)+16 G^{2} \omega_{0}\right]\right]^{\frac{1}{2}}\right] \\
& \simeq \frac{1}{2}\left[\left(\omega_{0}^{2}+\omega_{e x c}^{2}\right) \pm\left|\omega_{0}^{2}-\omega_{e x c}^{2}\right|\right. \\
& \left.+r_{0}^{2}\left[C(\bar{\omega}) \lambda_{0} \pm \frac{\lambda_{0}}{\left|\omega_{0}^{2}-\omega_{e x c}^{2}\right|}\left[C(\bar{\omega})\left(\omega_{e x c}^{2}-\omega_{0}^{2}\right)+8 G^{2} \omega_{0}\right]\right]\right]
\end{aligned}
$$

where the short notation $C(\bar{\omega})=\left(2 \Delta-\lambda_{0}\right) \alpha(\omega=\bar{\omega})$ was introduced and the result was expanded at first order in $r_{0}^{2}$. $\bar{\omega}$ assumes the values $\omega_{\text {exc }}$ or $\omega_{0}$ corresponding to the zeroth order term in the expansion of $\omega$ in $r_{0}^{2}$.

Two cases can be distinguished in Eq.(42): $\omega_{\text {exc }}>\omega_{0}$, the more physical one, and $\omega_{\text {exc }}<\omega_{0}$ which can only occur close to the metal-charge-transfer-insulator transition, where the exciton mode completely softens [15], [37]. In the former case one has

$$
\begin{aligned}
& \omega^{2}=\omega_{e x c}^{2}+r_{0}^{2} \lambda_{0}\left(C\left(\omega_{e x c}\right)+\frac{4 G^{2} \omega_{0}}{\omega_{e x c}^{2}-\omega_{0}^{2}}\right) \\
& \omega^{2}=\omega_{0}^{2}-r_{0}^{2} \lambda_{0} \frac{4 G^{2} \omega_{0}}{\omega_{e x c}^{2}-\omega_{0}^{2}}
\end{aligned}
$$

whereas in the latter case the modes are

$$
\begin{aligned}
& \omega^{2}=\omega_{\text {exc }}^{2}+r_{0}^{2} \lambda_{0}\left(C\left(\omega_{\text {exc }}\right)-\frac{4 G^{2} \omega_{0}}{\omega_{0}^{2}-\omega_{\text {exc }}^{2}}\right) \\
& \omega^{2}=\omega_{0}^{2}+r_{0}^{2} \lambda_{0} \frac{4 G^{2} \omega_{0}}{\omega_{0}^{2}-\omega_{\text {exc }}^{2}} .
\end{aligned}
$$

It should be noted that in both cases a "level repulsion" occurs so that the higher energy mode is pushed at even higher energy, while the lower energy one is made softer by the reciprocal interaction. However, it is important to stress the fact that this $q=0$ analysis does not reveal any complete softening of the collective modes in the limit of vanishing $r_{0}^{2}$, where the energies of the modes reduce to their "bare" values $\omega_{\text {exc }}$ and $\omega_{0}$. This indicates that any occurrence of $q=0$ instabilities in the small $r_{0}$ region of the system is not due to a softening of the modes. Of course the above analysis was confined to the $q=0$ case and, 
therefore, it does not allow to draw any conclusion on the occurrence of a collective mode softening at a finite wavelength or at large $r_{0}$. The investigation of this possibility requires a numerical analysis which was indeed performed. The result is that the static susceptibilities diverge first at $q=0$ and the softening of the modes at finite $q$ only takes place inside an unstable region, characterized by $F_{0}^{s}<-1$. This instability occurs as a consequence of the repulsion vs attraction unbalance generally presented in Section II, which we now elucidate for the specific three-band model under consideration.

\section{B. The Landau Fermi-liquid analysis}

In order to investigate the instability conditions of the model we now turn to a Fermiliquid analysis transposing our $1 / N$ calculation to the standard formalism presented in Section II and identifying the Landau amplitudes at leading order in $1 / N$. In particular, starting from the definition (28) of the singlet effective scattering amplitude one can introduce the standard Landau amplitudes

$$
\Gamma_{\omega}\left(k, k^{\prime}\right)=-\lim _{\omega \rightarrow 0} \lim _{q \rightarrow 0} \sum_{\mu, \nu} \tilde{\Lambda}_{11}^{\mu}\left(k^{\prime},-q\right) D^{\mu \nu}(q, \omega) \tilde{\Lambda}_{11}^{\nu}(k, q) .
$$

To carry out an analytical treatment we again assume $t_{p p}=0$. Then, since the model only includes a direct copper-oxygen transfer integral, $\Gamma_{\omega}\left(k, k^{\prime}\right)$ depends on $k$ and $k^{\prime}$ only via $\gamma_{k}$ and $\gamma_{k^{\prime}}$. Therefore, by taking the quasiparticles at the Fermi surface (where $\gamma_{k}=$ constant $=$ $\gamma_{F}$ ), only the "zeroth" harmonic would be non zero and given by

$$
\Gamma_{\omega}=-\lim _{\omega \rightarrow 0} \lim _{q \rightarrow 0} \sum_{\mu, \nu} \tilde{\Lambda}_{11}^{\mu}\left(k_{F},-q\right) D^{\mu \nu}(q, \omega) \tilde{\Lambda}_{11}^{\nu}\left(k_{F}, q\right) .
$$

To explicitly get the expression of $\Gamma_{\omega}$ we first calculate

$$
\begin{aligned}
& \Gamma(\omega)=-\frac{1}{2 N r_{0}^{2}}\left(\begin{array}{c}
-\frac{R_{k_{F}}^{2}-\Delta^{2}}{2 R_{k_{F}}} \\
i \frac{R_{k_{F}}+\Delta}{2 R_{k_{F}}} \\
-2\left(G_{d} u_{k_{F}}^{2}+G_{p} v_{k_{F}}^{2}\right)
\end{array}\right) \\
& \times\left(\begin{array}{ccc}
a & -i-i a\left(\Delta-\lambda_{0}\right) & 2 r_{0} a h(\omega) \\
-i-i a\left(\Delta-\lambda_{0}\right) & \lambda_{0}-\left(\Delta-\lambda_{0}\right)^{2} a & -2 i r_{0}^{2}\left(\Delta-\lambda_{0}\right) a h(\omega) \\
2 r_{0} a h(\omega) & -2 i r_{0}^{2}\left(\Delta-\lambda_{0}\right) a h(\omega) & r_{0}^{2} \omega_{0}\left(1+4 r_{0}^{2} a h(\omega)\right) /\left(\omega_{0}^{2}-\omega^{2}\right)
\end{array}\right) \\
& \times\left(\begin{array}{c}
-\frac{R_{k_{F}}^{2}-\Delta^{2}}{2 R_{k_{F}}} \\
i \frac{R_{k_{F}}+\Delta}{2 R_{k_{F}}} \\
-2\left(G_{d} u_{k_{F}}^{2}+G_{p} v_{k_{F}}^{2}\right)
\end{array}\right)
\end{aligned}
$$

with $a \equiv a(\omega) \equiv I(\omega)\left[1-\left(2 \Delta-\lambda_{0}+8 r_{0}^{2} V\right) I(\omega)\right]^{-1}$ and $h(\omega)=\omega_{0} G^{2} /\left(\omega_{0}^{2}-\omega^{2}\right)$ This gives

$$
\Gamma(\omega)=\Gamma_{0}+a(\omega) \Gamma_{1}(\omega),
$$

with 


$$
\begin{aligned}
N \Gamma_{0} & =\frac{1}{8 r_{0}^{2}}\left(1+\frac{\Delta}{R_{k_{F}}}\right)^{2}\left(2 R_{k_{F}}-2 \Delta+\lambda_{0}\right)-\frac{2 \omega_{0}}{\omega_{0}^{2}-\omega^{2}}\left(G_{d} u_{k_{F}}^{2}+G_{p} v_{k_{F}}^{2}\right)^{2} \\
N \Gamma_{1} & =-\frac{1}{2 r_{0}^{2}}\left[\frac{1}{2}\left(1+\frac{\Delta}{R_{k_{F}}}\right)\left(R_{k_{F}}-2 \Delta+\lambda_{0}\right)\right. \\
& \left.+4 r_{0}^{2} \frac{2 \omega_{0}}{\omega_{0}^{2}-\omega^{2}}\left(G_{d}-G_{p}\right)\left(G_{d} u_{k_{F}}^{2}+G_{p} v_{k_{F}}^{2}\right)\right]^{2} .
\end{aligned}
$$

A simple expression for $\Gamma_{\omega}$ can be obtained taking the $\omega \rightarrow 0$ limit of the above formulas in the small $r_{0}$ limit. It is worth noting that, whereas $a(\omega)$ is always finite, both $\Gamma_{0}$ and $\Gamma_{1}$ grow very large when $r_{0} \rightarrow 0$. This is reminiscent of the infinite $\mathrm{U}$ we started with. However a straightforward expansion of Eqs.(50) and (51) shows that the leading terms of order $1 / r_{0}^{2}$ (which characterize the model even in the absence of the e-ph interaction) cancel and only finite contributions are left. Therefore in this model a finite effective scattering amplitude is the result of a cancellation between a very large bare repulsion and a very large attraction due to interband screening, to which a finite phonon contribution is added.

The resulting dynamical scattering amplitude is, for $r_{0} \rightarrow 0$

$$
N \Gamma_{\omega} \approx \frac{4 \lambda_{0}}{\left(\lambda_{0}-\Delta\right)^{2}}\left[2 t_{p d}^{2}\left(\frac{\gamma_{4}}{\gamma_{2}}-1+\frac{\Delta^{2}}{4 t_{p d}^{2} \gamma_{2}}\right)-\frac{\left(\lambda_{0} G_{p}-\Delta G_{d}\right)^{2}}{2 \lambda_{0} \omega_{0}}\right]
$$

The first term represents the purely electronic (interband screened) repulsive contribution $\Gamma_{\omega}^{e}$ to $\Gamma_{\omega}$ [15], whereas the second term is the attraction, which arises from the e-ph interaction. In particular it would coincide with the second term in the r.h.s. of Eq.(12), if the simple case $G_{p}=G_{d}=g$ is considered and the identification $\gamma^{2} \equiv g^{2} / \omega$ is made [38]. One can easily check that the e-ph vertex corrections in Eq.(52) (i.e. the factors $\Lambda_{\alpha} \Lambda_{\beta}$ with $\alpha, \beta=p, d$, which multiply $\left.G_{\alpha} G_{\beta} / \omega_{0}\right)$ can be written as

$$
\Lambda_{\alpha}^{\omega}=\frac{\mathrm{d}}{\mathrm{d} \varepsilon_{\alpha}^{0}} G_{\mathrm{qp}}^{-1}\left(k_{F}, \omega=0\right),
$$

where $G_{\mathrm{qp}}(k, \omega)=\left(\omega-E_{k}\right)^{-1}$ is the quasiparticle Green function and the derivatives are taken at fixed doping $\delta$. One can then discover that in a multiband model the vertex corrections are still different from zero in the dynamical limit (cf. Eq.(1i) with $z_{w}=1$ because we are considering quasiparticles) and in fact can act to enhance the vertices. For instance, at $\delta=0^{+}$, we obtain $\Lambda_{p}^{\omega}=\lambda_{0} /\left(\lambda_{0}-\Delta\right)$, which reduces to unity only in the $\lambda_{0} \rightarrow \infty$ limit, i.e. $\varepsilon_{p}^{0}-\varepsilon_{d}^{0} \rightarrow \infty$. To obtain the vertex correction in the static limit we need to evaluate $\frac{\mathrm{d}}{\mathrm{d} \varepsilon_{\alpha}^{0}} G_{\mathrm{qp}}^{-1}\left(k_{F}, \omega=0\right)$ at fixed chemical potential [39]. We obtain

$$
\Lambda_{\alpha}^{q}=\frac{\Lambda_{\alpha}^{\omega}}{1+N \nu^{*} \Gamma_{\omega}^{e}}
$$

where $\Gamma_{\omega}^{e}$ is the first term in the r.h.s. of Eq.(52). Eq.(54) shows the dramatic difference between the dynamic and static limits. As discussed in Section II, this is a generic feature of strongly correlated systems, which one has to take into account since it can strongly affect the relevance of the e-ph coupling 40. 


\section{The phase diagram}

The previous analysis of the restricted model with $t_{p p}=0$ provides valuable informations on the possible occurrence of instabilities of the Fermi liquid. In particular, having found $\Gamma_{\omega}$, one can determine the Landau parameter $F_{0}^{s} \equiv N \nu^{*} \Gamma_{\omega}$. Since the criterion for a finite positive compressibility is $F_{0}^{s}>-1$, and since $\nu^{*} \propto \frac{1}{r_{0}^{2}} \gg 1$ in the small doping region, close to the insulating region $\left(\varepsilon_{p}^{0}-\varepsilon_{d}^{0}>3.35 t_{p d}\right)$ the stability criterion reduces to $\Gamma_{\omega}>0$. Taking into account the self-consistency condition $\lambda_{0}=4 t_{p d}^{2} \gamma_{2} / \Delta$ one is lead to the following inequality

$$
\left(\lambda_{0} G_{p}-\Delta G_{d}\right)^{2}<4 t_{p d}^{2} \lambda_{0} \omega_{0}\left(\frac{\gamma_{4}}{\gamma_{2}}-1+\frac{\Delta}{\lambda_{0}}\right)
$$

Even for a weak e-ph coupling, this condition can easily be violated (see below).

A clearer understanding of the stability condition can be obtained by considering the phonon coupled separately with the copper or the oxygen local density. In the case of $G_{d}=0$ the condition (55) becomes

$$
\frac{G_{p}^{2}}{\omega_{0}}<\frac{4 t_{p d}^{2}}{\lambda_{0}}\left(\frac{\gamma_{4}}{\gamma_{2}}-1+\frac{\Delta}{\lambda_{0}}\right)
$$

whereas for $G_{p}=0$ one has

$$
\frac{G_{d}^{2}}{\omega_{0}}<\frac{\lambda_{0}^{2}}{\Delta^{2}} \frac{4 t_{p d}^{2}}{\lambda_{0}}\left(\frac{\gamma_{4}}{\gamma_{2}}-1+\frac{\Delta}{\lambda_{0}}\right) .
$$

Then one can easily recognize that, in the positive doping case with $\lambda_{0}>\Delta$, the coupling of the phonon with the oxygen holes $G_{p}$ is more effective in driving the instability. The role of $\lambda_{0}$ and $\Delta$ is interchanged at negative doping so that $\lambda_{0}<\Delta$ and in this latter case the coupling $G_{d}$ plays a major role in rendering the Fermi liquid unstable. Notice, however, that the model itself is not particle-hole symmetric so that the expressions (56) and (57) do not interchange in going from negative to positive doping.

It is worth noting that, no matter how small the e-ph coupling is or how large the phonon frequency is, the instability will always take place by increasing the large bare charge-transfer difference $\left(\varepsilon_{p}^{0}-\varepsilon_{d}^{0}\right) / t_{p d}$. This is due to the fact that, for large $\left(\varepsilon_{p}^{0}-\varepsilon_{d}^{0}\right) / t_{p d}$ one has $\lambda_{0} \gg \Delta$ for positive doping or $\lambda_{0} \ll \Delta$ for negative doping. Then it is possible to make the r.h.s. of Eqs.(56) or (57) smaller than the l.h.s..

The estimates (56),(57) were derived for the three-band Hubbard model with $t_{p p}=0$ close to the insulating regime, where $r_{0} \approx 0$. To investigate the finite-doping regime and the model with finite oxygen-oxygen hopping we explicitly carried out a leading order $1 / \mathrm{N}$ analysis of the static $q$-dependent density-density response function $\chi(q, \omega=0) \equiv<\left(n_{p}(q)+\right.$ $\left.n_{d}(q)\right)\left(n_{p}(-q)+n_{d}(-q)\right)>$, which gives the compressibility of the system once the $q \rightarrow 0$ limit is taken. This response function is obtained as a linear combination of the orbital densitydensity correlation functions in Eq.(30). A divergent $\chi(q, \omega=0)$ signals an instability of the Fermi liquid occurring at a wavevector $q$.

We performed the calculations for many values of the parameters, and specifically for various phonon frequencies $\omega_{0}$ and various e-ph couplings $G_{p}$ and $G_{d}$, and we particularly 
explored the range of parameters, which could be relevant for the copper oxides. According to recent estimates [43], [44] we considered various values of $\omega_{0}$ ranging in the interval between 0.01 and $0.08 \mathrm{eV}$, particularly focusing on the $0.02 \mathrm{eV}$ region, where the phonon density of states is highest. As far as the e-ph couplings are concerned, these quantities are not directly accessible in experiments and their theoretical calculation is not easy due to the strongly interacting nature of the high temperature superconductors and to the nesting occurring in their Fermi surface [45]. This is why we explored many possible cases obtaining qualitatively similar results. A reasonable choice is to use both $G_{p}$ and $G_{d}$ different from zero, because a coupling of the lattice with both oxygen and copper hole density is naturally expected. The values of $G_{d}$ and $G_{p}$ were such that a reasonable effective coupling $\lambda<1$ results far enough from the instability region. Moreover the choice $G_{p}>G_{d}$ appears reasonable in the case of intermediate hole doping according to the observations reported in the paragraph after Eq.(17): i) $G_{p}$ is proportional to $\left\langle n_{d}\right\rangle$, while $G_{d}$ is proportional to $\left\langle n_{p}\right\rangle$, with $\left\langle n_{d}\right\rangle$ usually being larger than $\left\langle n_{p}\right\rangle$; ii) a factor $1 / \sqrt{M_{O}}$ is included in the definition of $G_{p}$, whereas an analogous factor $1 / \sqrt{M_{C u}}$ enters in $G_{d}$ further justifying the assumption $G_{p}>G_{d}$, since $M_{C u}>M_{O}$. Both theoretical calculations and experiments in superconducting cuprates [1] support the above qualitative arguments. The fact that oxygen holes are more strongly coupled to the lattice than holes on copper has relevant physical consequences in the light of the above observation that, for positive doping, $G_{p}$ is more effective than $G_{d}$ in driving the instability.

The phase diagrams in the $\left(\varepsilon_{p}^{0}-\varepsilon_{d}^{0}\right) / t_{p d}$ vs $\delta$ plane resulting from the analysis of $\chi(q, \omega=$ $0)$ are shown in Figs. 1 and 2 for typical parameter sets. The instability line indicates where $\chi(q \rightarrow 0, \omega=0)$ diverges and it delimits a region of negative compressibility. The most impressive consequence of the coupling with the phonons is that a large unstable region appears at large doping or at large bare charge-transfer gap in both diagrams. It is worth noting again that the instability is not related to any collective mode softening. It can rather be interpreted as due to the attraction arising from the e-ph interaction eventually resulting in the overdamping of the zero-sound mode. Approaching the instability, the zerosound mode first enters the continuum and gets Landau-damped. Eventually it becomes overdamped and the instability takes place.

It is important to stress that the instability of the phase diagrams in Figs. 1 and 2 is an electronic instability even if it is driven by the coupling with the lattice. Moreover we find that the instability of $\chi(q, \omega=0)$ first occurs at $q \rightarrow 0$ and is therefore a signature of a longwavelength, static, thermodynamical PS region to be identified by a Maxwell construction. To make this point more explicit, we report in Fig.3 the Cooper-channel static scattering amplitude between quasiparticles on the Fermi surface $\Gamma^{C}\left(k_{F}, k_{F}^{\prime}, \omega=0\right)$ (see Eq.(29) and cf. also the $\omega=0$ limit of Eq.(13) $)$ for a system with finite $t_{p p}\left(=0.2 t_{p d}\right)$ and at various dopings. According to the observation already reported at the beginning of Subsection A, the scattering amplitude carries information on the instability because a divergency in this quantity can only be due to a divergency in the boson propagator also entering the expression for $\chi(q, \omega=0)$, with $q=k_{F}-k_{F}^{\prime}$. Thus a divergent scattering amplitude directly signals a divergent $\chi(q, \omega=0)$. As it can be seen in Fig.3, the divergency can also occur at sizable $q$ 's inside the unstable region. This can have relevant physical consequences, once a longrange Coulomb force is considered in the model. This latter interaction would stabilize the system in the regions of the phase diagram where $\chi(q, \omega=0)$ shows divergencies at low- $q$. 
This would prevent the occurrence of PS, but would leave open the possibility of finite- $q$ instabilities: Most probably a sizable unstable region would survive to the introduction of a long-range Coulomb repulsion in the phase diagram parts where $\chi(q, \omega=0)$ was diverging at sizable $q$ 's, i.e. the divergency of the density-density response function would first occur at a finite $q$ leading to the formation of incommensurate CDW. A similar phenomenon was first suggested for a different model in Refs. [13]- [15] and seems to be confirmed by a static analysis of a pseudospin model in Ref. [20]. Of course the above argument misses the dynamical aspects of the problem, and should be taken as purely indicative of a possible scenario. One should also take into account that superconductivity can take place near the instability line where $F_{0}^{s}=-1$ and act to stabilize the system (i.e. superconductivity could partially or fully preempt the instability region).

Few comments on the difference between the two phase diagrams of Figs. 1 and 2 are in order. It is apparent that the prominent effect of the direct oxygen-oxygen hopping is a stabilization at low doping and large $\varepsilon_{p}^{0}-\varepsilon_{d}^{0}$. In order to clarify this point we performed a calculation of the compressibility both in the presence and in the absence of $t_{p p}$ in the limit of very large $\left(\varepsilon_{p}^{0}-\varepsilon_{d}^{0}\right) / t_{p d}$ and small positive doping. Due to the slave-boson-meanfield band renormalization the lowest quasiparticle band closely approaches the bottom of the intermediate band, mostly of oxygen character. This latter band is a flat non-bonding oxygen band in the absence of $t_{p p}$ and acquires a dispersion $4 t_{p p}$ in the presence of direct oxygen-oxygen hopping if the mixing with the copper $d_{x^{2}-y^{2}}$ orbitals is neglected. In the $\left(\varepsilon_{p}^{0}-\varepsilon_{d}^{0}\right) / t_{p d} \rightarrow \infty$ and $\delta \rightarrow 0^{+}$one has a vanishing distance between the renormalized $d$ atomic level and the bottom of the pure-oxygen band $\tilde{\Delta} \equiv \varepsilon_{p}^{0}-4 t_{p p}-\varepsilon_{d} \rightarrow 0$. In this limit the calculation greatly simplifies and, for $t_{p p} \neq 0$ the compressibility per spin assumes the form

$$
\begin{aligned}
& \kappa \propto \frac{1}{t_{p p}} \quad d=2 ; \\
& \kappa \propto \frac{1}{t_{p p}}\left(\frac{\tilde{\Delta}}{t_{p p}}\right)^{\frac{1}{2}} \quad d=3 .
\end{aligned}
$$

This result is suggestive of a decoupling between the $p$ and the $d$ levels occurring when $\left(\varepsilon_{p}^{0}-\varepsilon_{d}^{0}\right) / t_{p d} \rightarrow \infty$ : the compressibility for the interacting system of $\left(1+0^{+}\right)$holes in the mixed $p$ - $d$ quasiparticle band beares resemblance with the compressibility of a system of $\delta=$ $0^{+}$non-interacting holes in the pure-oxygen band. In this latter system the compressibility coincides with the density of states, which is of order $1 / t_{p p}$ and is independent on doping in two dimensions, whereas it vanishes with doping in the $d=3$ case. This is precisely the result reported in Eqs.(58) and (59) respectively, once one remembers that, in the $\left(\varepsilon_{p}^{0}-\varepsilon_{d}^{0}\right) / t_{p d} \rightarrow \infty$ and $\delta \rightarrow 0^{+}$limits, $\tilde{\Delta}$ is vanishingly small.

Eqs.(58) and (59) also express the fact that in the large $\left(\varepsilon_{p}^{0}-\varepsilon_{d}^{0}\right) / t_{p d}$ and low-doping limit, the compressibility of the system diverges when $t_{p p}$ tends to zero (even for the three-band Hubbard model in the absence of e-ph coupling). Since $\kappa^{e}=\nu^{*} /\left(1+N \nu^{*} \Gamma_{\omega}^{e}\right)$, this result can be rephrased as $\Gamma_{\omega}^{e} \simeq t_{p p}$. Thus, when $t_{p p} \rightarrow 0$ and the quasiparticle density of states $\nu^{*}$ is large, even a very small attraction leads to a negative $\Gamma_{\omega}$, immediately resulting in a violation of the Landau stability condition $F_{0}^{s}=N \nu^{*} \Gamma_{\omega}<-1$. The three-band Hubbard model with $t_{p p}=0$ and $\varepsilon_{p}^{0}-\varepsilon_{d}^{0}=\infty$ case is strictly analogous to the $U=\infty$ single-band case [18]. This clarifies why, the region with infinite compressibility at low doping and large 
$\varepsilon_{p}^{0}-\varepsilon_{d}^{0}$ in the phase diagram of the system of Fig.1 $\left(t_{p p}=0\right)$, is more extended than the one for the system of Fig.2.

\section{The Cooper instability}

Finally we investigated the possibility of Cooper pairing. As already pointed out, superconductivity can appear as a precursor of the PS instability. In particular a simple argument can be put forward [13], [14] suggesting that a region of large compressibility is a good candidate in order to find superconductivity. In the general language of Landau Fermi-liquid theory the compressibility can be written as $\kappa=\nu_{0} \frac{m^{*}}{m} /\left(1+F_{0}^{s}\right)$. Then, if the compressibility gets large without being accompanied by a large mass enhancement, (as it happens near the instability line), this means that the denominator is becoming small indicating a negative $F_{0}^{s}$ (indeed $F_{0}^{s}=-1$ at the instability line). Although this parameter is related to the quasiparticle scattering in the particle-hole channel, it seems reasonable to expect attraction in the particle-particle channel as well. According with this plausibility argument and according to the previous experience in other strongly interacting models, we therefore investigated the Fermi surface average of the particle-particle scattering amplitude defined in Eq.(29)

$$
\lambda_{l}=-\frac{\iint d k d k^{\prime} \delta(E(k)-\mu) \delta\left(E\left(k^{\prime}\right)-\mu\right) g_{l}(k) \Gamma^{C}\left(k, k^{\prime} ; \omega=0\right) g_{l}\left(k^{\prime}\right)}{\int d k \delta(E(k)-\mu) g_{l}(k)^{2}} .
$$

with $g_{s_{1}}(k)=\cos \left(k_{x}\right)+\cos \left(k_{y}\right), g_{s_{2}}(k)=\left(\cos \left(k_{x}\right)-\cos \left(k_{y}\right)\right)^{2}, g_{d_{1}}(k)=\cos \left(k_{x}\right)-\cos \left(k_{y}\right)$ and $g_{d_{2}}(k)=\sin \left(k_{x}\right) \sin \left(k_{y}\right)$ projecting the interaction onto the s-wave and d-wave channels. (Notice that $\lambda_{l}>0$ means attraction). Whereas the couplings $\lambda_{d}$ are found to be generally attractive near (and inside) the unstable region, we find s-wave Cooper instabilities only very close to the instability line. The results are tabulated at various doping concentrations for the case with $t_{p p}=0$ and $\left(\varepsilon_{p}^{0}-\varepsilon_{d}^{0}\right) / t_{p d}=3.3 t_{p d}$ in Table 1 and for the case with $t_{p p}=0.2 t p d$ and $\left(\varepsilon_{p}^{0}-\varepsilon_{d}^{0}\right) / t_{p d}=4.9 t_{p d}$ in Table 2. In both cases the phonon frequency and the e-ph couplings are $\omega_{0}=0.02 t_{p d}$, and $G_{p}=0.15 t_{p d}$, and $G_{d}=0.1 t_{p d}$, respectively. With the set of parameters related to Table 1 the critical doping for the occurrence of the instability is $\delta_{c}=0.21$, whereas for the set of Table 2 the instability is at $\delta_{c}=0.23$. Our analysis indicates the sure existence of d-wave pairing in sizable regions of model (21) in the limit of large-N near the instability, whereas the occurrence of s-wave pairing takes place in a much narrower region [41]. However, it should be emphasized that the presence of a s-wave static Cooper instability only in a narrow region, by no means excludes the possibility of having s-wave superconductivity in a much larger area of our phase diagram. Only an appropriate Eliashberg dynamical analysis can allow to draw a conclusion, specially in the light of the considerations on the strong frequency dependence of the vertex corrections discussed in Section II. Of course the same applies to the attraction in the d-wave channels, which could also be greatly favored by dynamical effects. The full Eliashberg dynamical analysis unfortunately involves a difficult complete treatment of the momentum and frequency dependence of the effective scattering amplitude, which is beyond the scope of the present paper. Nevertheless valuable informations can be gained from an analysis of the e-ph vertex. The aim of this analysis is to provide useful quantitative indications on the behaviour of the e-ph interaction as a function of both momentum and imaginary frequency in various parts of the phase 
diagram as a preliminary step towards the implementation of the full Eliashberg treatment of the effective phonon-mediated e-e scattering amplitude. Also in this case we checked that our results do not qualitatively change by varying the model parameters. In particular we choose the values of the various quantities in order to allow for a possible connection with the experimentally known features of the copper oxides. We choose the value of the bare atomic level difference $\varepsilon_{p}^{0}-\varepsilon_{d}^{0}=4.9 t_{p d}$ so that a CT optical gap in the insulating phase of about $2 t_{p d}$ would result. Assuming the usual value of $t_{p d}=1.3 \mathrm{eV}$ for the copper-oxygen hopping integral, this latter value turns out to be $2.6 \mathrm{eV}$, and is not much larger than the experimentally known values of the $\mathrm{CT}$ gap in the insulating phase of the superconducting copper oxides (e.g. in Ref. 42] the reported value for $\mathrm{La}_{2} \mathrm{CuO}_{4}$ is $2.3 \mathrm{eV}$ ). As values of the phonon frequency we take $0.02 t_{p d} \simeq 0.026 \mathrm{eV}$ and $0.08 t_{p d} \simeq 0.104 \mathrm{eV}$. The first value was chosen because in this frequency region the experimental phononic density of states is large, whereas the larger value was used to extend the region where dynamical effects can take place before the phonon frequency cuts off the effective interaction. The value of the e-ph couplings was chosen accordingly in order to obtain reasonable values of $\lambda$ 45.

We first report in Fig.5 the interaction (slave-boson) renormalized vertex between phonons and quasiparticles without phonon processes included in it (see Fig.4) as a function of the Matsubara frequency. Two of these quantities can be joined to a bare phonon propagator to give the one-phonon effective scattering amplitude specializing to the three-band Hubbard model the general expression (3).

On the other hand, joining two of the above vertices with a fully renormalized phonon propagator leads to the full phonon-mediated scattering amplitude in the Cooper channel. This quantity was represented by the second term of Eq.(13) in Section II and is reported in Fig.6 for the three-band Hubbard model as a function of the Matsubara frequency. The momenta of the external fermions correspond to the Cooper channel with $k$ and $k^{\prime}$ on the Fermi surface.

It is clear that the results reported in Figs. 5 and 6 quantitatively confirm the general qualitative analysis of Section II. Fig. 5 displays the strong dependence of the one-phonon vertex from the momentum vs frequency ratio. A typical bandwidth of about $0.2 t_{p d}$ was chosen, giving a Fermi surface density of states $\approx 13 / t_{p d}$. We only report the results corresponding to a doping $\delta=0.225$ since no significant dependence of this vertex from the doping is detected, so that the closeness to the instability is immaterial in this quantity. On the other hand, it is apparent that a rapid increase occurs in the phonon-quasiparticle vertex, when the frequency becomes larger than $v_{F} q$. This is most evident at low momenta, where the phonon-quasiparticle vertex $G_{\text {eff }}$ increases fast by more than one order of magnitude, from the small (see inset in Fig.5) value at zero frequency up to a sizable value at large-frequency. This latter is much larger because at frequency larger than the bandwidth for quasiparticle-quasihole excitations $\left(v_{F} q\right.$ at small $q$ in an isotropic system) the intraband electronic screening is ineffective (cf. Eq.(11)) [46].

Analyzing in detail the contributions coming from the vertex corrections, we discovered that in this "high"-energy region the interband screening acts to enhance the vertex at small $q$ 's by a factor of about 1.5 with respect to the bare vertex $\left(G_{0} \simeq 0.2\right)$. The bare value is only recovered at very high energy (much larger than the interband CT energy $\Delta$ ), and/or large $q$.

More dramatic are the momentum and doping dependences of the attractive scattering 
amplitude in Fig.6. In the stable region $(\delta=0.2)$, a maximum in the attraction is observed at finite frequency and low momentum. This maximum is a result of the balance between the energy dependence of the vertex correction and of the boson propagator (see the inset). The interaction at low momenta is by far larger than the attraction at large momenta. The same behavior as a function of momenta holds near the unstable region of doping, $(\delta=0.225)$, but at low energy and small momentum the attraction is not suppressed by vertex corrections, because in the vicinity to the instability line the attraction reaches its maximum at $\omega=0$.

Finally Figs.7 and 8 report the total effective scattering amplitude in the Cooper channel $\Gamma^{C}\left(k_{F}, k_{F}^{\prime}, \omega_{n}\right)$ (continuous lines) resulting from the sum of the attractive amplitude (dotted lines) of Fig.6 and the repulsion arising from purely electronic scattering processes (dashed lines) as a function of Matsubara frequencies at small (a) and large $q$ (b).

Being the calculations performed close to the instability, we see that the e-ph interaction gives a large contribution to the total interaction at small $q$, leading to an overall attraction at low frequencies. On the contrary, at large $q$ the full interaction is only governed by the pure e-e repulsion and no phonon energy scale is detectable in its frequency dependence. Fig.8 reports the same quantities of Fig.7, but for a larger phonon frequency (consequently $G_{p}$ has been increased, $G_{p}=0.3 t_{p d}$, to drive the system unstable at reasonable doping $\left.\delta_{c}=0.185\right)$. Qualitatively the results of Fig.8 show the same behaviour depicted in Fig.7, but it is evident that the phonon-mediated attraction extends over a larger frequency range. We also found that in this latter case the attraction persists up to larger momenta. At large $q$ (Fig.8b) the results for $\Gamma^{C}\left(k_{F}, k_{F}, \omega_{n}\right)$ are still very similar to those of Fig. $7 \mathrm{~b}$.

Finally Fig.9 reports $\Gamma^{C}\left(k_{F}, k_{F}^{\prime}, \omega_{n}\right)$ as well as its attractive and purely electronic repulsive components at $\delta=0.2$. Due to the depressing effect of the vertex at low frequencies, the phonon contributes very little to $\Gamma^{C}\left(k_{F}, k_{F}^{\prime}, \omega_{n}\right)$ even at small $q$. Nevertheless, as it is also apparent from Fig.6, at frequency of the order of $\omega_{0}$ the attractive part contributes sizably to the total interaction. This clearly indicates that at small q, contrary to the static attraction, which is strongly suppressed by the vertex corrections, the finite frequency attraction persists in regions of doping that are far from the instability line.

The analysis summarized in Figs.5-9 is of obvious pertinence in a complete Eliashberg treatment of the superconductivity problem. In particular it is evident that the huge enhancement of the attractive part of the scattering amplitude near the instability line can be responsible for large critical temperatures despite the small e-ph coupling. The closeness to a PS instability appears therefore as a favorable condition in order to obtain high temperature superconductivity from a phonon-mediated attraction similarly to what suggested in the context of purely electronic pairing mechanisms.

\section{CONCLUSIONS}

In the present work we investigated the e-ph interaction in the presence of a strong local repulsion within a general Landau Fermi-liquid framework. Using standard Ward identities, we pointed out the strong dependence of the e-ph vertex on the momentum vs frequency ratio in a strongly interacting system displaying a large effective-mass enhancement, but not too large compressibility. In particular we showed that the dimensionless attractive quasiparticle scattering mediated by a single phonon exchange coupled to the electronic density is strongly suppressed by vertex corrections due to the e-e repulsion when $v_{F} q>\omega$. On the 
contrary the dimensionless effective one-phonon-mediated attraction is strongly enhanced by the effective mass increase when $v_{F} q<\omega$. These results stay valid within a random-phase resummation of phonon exchanges, provided the system is far from an instability. This strong momentum vs frequency dependence of the e-ph coupling shows up in different ways in different physical quantities. In particular it was found in a three-band Hubbard model with intersite "covalent" e-ph coupling [17], that phonons contribute little to the quasiparticle scattering as far as transport properties are concerned. We expect a similar depression for the e-ph coupling considered in this paper [49]. This is so because transport properties involve low-frequency-high-momentum processes, where the large-momentum limit of the e-ph interaction is mostly involved. In this limit the e-ph interaction is strongly suppressed, particularly in the low-doping regime, where the effects of the strong e-e interaction play a major role. A different behaviour is expected in other quantities where large frequencies are more relevant. This is in agreement with recent calculations [18 performed in a singleband Hubbard model with an on-site "ionic" e-ph coupling, which show, indeed, that the Eliashberg function $\alpha^{2} F(\omega)$ determining superconductivity, is much less reduced than the analogous quantity determining the transport properties.

These remarks are obviously relevant in any dynamical analysis of the pair formation in strongly interacting systems like, e.g., high temperature superconducting cuprates, fullerenes or $\mathrm{Ba}_{1-\mathrm{x}} \mathrm{K}_{\mathrm{x}} \mathrm{BiO}_{3}$. In particular, our work calls for a critical reanalysis of effective potential models in the Eliashberg approach of pairing [2] [50], when a strong e-e interaction is present in the systems. Most of the work in this field has, in fact, been carried out using model potentials, where the momenta are averaged on the Fermi surface. This leads to a trivial momentum dependence (likely overestimating the role of large momenta), which misses the peculiar strong momentum vs frequency dependence revealed in the interacting systems.

On the other hand our general analysis showed that the e-ph coupling can drive the strongly correlated system to an instability. This was specifically shown for the three-band Hubbard model in the $U \rightarrow \infty$ limit, where more specific features arise. In particular it was evidenced that the Fermi-liquid system has diverging long-wavelength density fluctuations in some regions of the $\left(\varepsilon_{p}^{0}-\varepsilon_{d}^{0}\right) / t_{p d}$ vs. $\delta$ phase diagram, even with a reasonably small e-ph coupling. This is due to the phonon induced attraction leading to the violation of the Landau stability criterion $F_{0}^{s}>-1$. In analogy with other cases [13]- [15] this appears as an overdamping of the zero-sound mode. Moreover it was found that superconducting pairing both in the s- and d-wave channels occurs close to the instability region. It is worth stressing that the detected Cooper instabilities occur in the presence of an infinite local repulsion $U$.

These findings are of obvious theoretical relevance in the context of high temperature superconductivity. The general considerations of Section II show that one is not allowed to draw any naive conclusion on the extension of the superconducting region on the basis of a static analysis, which neglects the presence of the e-e interaction in the e-ph coupling, once strong correlations are present. However, it should be noticed that the results of Section II indicate that, if a system displays Cooper phenomenon in a static $(\omega=0)$ analysis including vertex correction, the pairing can only be favoured by the extension of the analysis to finite frequencies. We also like to point out that phonon corrections to the e-ph vertex, which are usually neglected according to the Migdal theorem [47], have recently been considered in Ref. [48]. This analysis showed that these corrections, which are not negligible if the bandwidth vs phonon frequency ratio is sizable, tend to suppress the vertex in the large 
$v_{F} q / \omega$ region, whereas they tend to enhance the e-ph coupling when $v_{F} q / \omega$ is small. These corrections tend therefore to cooperate with the strong interaction effects discussed in the present paper.

Our results suggest that, if high temperature superconductivity is driven by e-ph coupling, this is possibly due to the fact that the system is close to a PS instability, where the argument of the vertex suppression does not apply. If this is the case, superconductivity itself would possibly provide the system a stabilizing mechanism with respect to superconductivity [11].

By increasing the bare e-ph coupling, one could indeed get a sizable attractive $\lambda_{s}(\omega=0)$ at small and intermediate doping if, for instance, the e-ph mediated attraction is evaluated at order $\gamma^{2}$ (a similar calculation has been carried out in Ref. [51] for the single-band infiniteU Hubbard model with a covalent (intersite) e-ph coupling). However, at the same time, $F_{0}^{s}$ (which is less affected by e-e vertex corrections), evaluated in the normal state, should be largely negative $\left(F_{0}^{s} \ll-1\right)$ and one should worry about the stability of the system. The straightforward approach would be to allow for pairing and then evaluate $\frac{\partial n}{\partial \mu}$ and then check for thermodynamic stability. This requires the evaluation of e-e screening effects in the superconducting state, where hopefully the (partial or full) opening of the gap will stabilize the system. In this paper we have kept the aptitude to evaluate the dimensionless couplings $\lambda_{l}$ in the stable region where $F_{0}^{s}>-1$, leaving to a future work the analysis of the region with $F_{0}^{s}<-1$ in the normal phase.

The generality of the arguments presented in Section II and the qualitative independence of the results obtained in the framework of the three-band Hubbard model by varying the eph couplings $G_{p}$ and $G_{d}$ and other parameters of the model (like, e.g., the phonon frequency $\omega_{0}$ or the oxygen-oxygen hybridization $t_{p p}$ ), witness that our results are rather robust and should also depend little on the particular choice of the phonon considered in the model. The choice taken in the model (17) was dictated by simplicity, but we do not expect qualitative differences in the physics of more realistic models. Besides superconductivity, our results provide a possible explanation for other general features of the superconducting cuprates as well. In particular our results concerning the presence of incommensurate CDW would explain the formation of the superstructures, which are present in many copper oxides, if this phenomenon has an electronic origin.

A relevant issue related to the work presented here concerns the possible formation of polarons in the three-band Hubbard model. The treatment of this long standing problem is beyond the scope of the paper, but it is worth emphasizing that the band narrowing occurring at low doping due to strong e-e repulsion could ease the polaron formation.

Some insight on this can be gained from the analysis of Monte Carlo calculations [52 showing that a polaronic regime may take place in a single-band model with one single electron coupled via a short-range interaction $g$ to optical phonons. The polaronic regime occurs as soon as the e-ph coupling $g$ exceeds a critical value $g_{c}$. The numerical analysis shows that the critical value depends on both the electronic bandwidth $(\sim t$ in Ref. [52]) and the phonon frequency $\left(\omega_{0}\right)$, and it can be represented by the condition $g_{c}^{2} /\left(t \omega_{0}\right)=\lambda_{c} \simeq 1$ in the adiabatic, $t \gg \omega_{0}$, regime or by the condition $g_{c} / \omega_{0}=\alpha_{c} \simeq 1$ in the antiadiabatic, $t \ll \omega_{0}$, regime.

The numerical Monte-Carlo analysis [52] only concerns the case of one single electron in a lattice, whereas the presence of other interacting electrons may modify the above picture. As 
discussed in Section II, the high sensitivity of the vertex corrections as a function of frequency and momentum renders the above conditions for polaron formation rather ambiguous, since it is not clear which frequency vs momentum regions dominate the e-e screening processes dressing the e-ph coupling $g$ (cf. Section II). A quick inspection to the $\left\langle c_{i}^{\dagger} c_{i}\left(a_{i}+a_{i}^{\dagger}\right)\right\rangle$ correlation function shows that a rather natural guess is that the relevant frequencies are smaller than the phonon frequency $\omega_{0}$. Then, if the quasiparticle bandwidth $t^{*}$ is larger than $\omega_{0}$, the vertex corrections to the phonon propagator are largely to be considered in the large momentum $\left(v_{F} q / \omega>1\right.$ and $\left.v_{F} q \approx t^{*}\right)$ and are large, thus leading to a suppression of the e-ph correlation. This would disfavor the establishment of a polaronic regime. On the other hand, as soon as the effective fermion bandwidth $t^{*}$ decreases (e.g. approaching the zero-doping insulating phase) the vertex corrections are in the high frequency region $\left(t^{*} \approx v_{F} q<\omega\right)$ and become small, so that the effective e-e coupling is given by $\lambda=g^{2} /\left(\omega t^{*}\right)$, which grows large. This increase likely leads to polaron formation. This considerations suggest that, if the e-e correlations do not lead to a fermion effective bandwidth smaller that the typical phonon frequencies, polaron are more difficult than in the non interacting system. On the contrary, if the closeness to an insulating phase reduces the effective bandwidth determining non-adiabatic conditions for the quasiparticles, then the polaronic regime is made easier: the e-e interaction itself reduces the electronic kinetic energy thus favoring the gain in lattice deformation energy related to the polaron formation. Notice that the lattice-driven instability of the Fermi liquid, which we showed to occur in the three-band Hubbard model for not too large e-ph coupling, does not appear to be related to the formation of polarons in the system. In fact the instability occurs at small $q$ 's (specifically at $q=0$ ), while the formation of local polarons involves all qs. Moreover the polaron instability is associated to the rapid softening and hardening of the phonon mode, while our dynamical analysis has shown that the instability is a consequence of an overdamping of the zero-sound, the phonon remaining massive.

A last remark can be made concerning the possible formation of bipolarons. In the limit of weak e-e repulsion $(U \simeq 0)$, and in the antiadiabatic regime, a large enough phonon-mediated attraction would lead to a violation of the Landau stability criterion $F_{0}^{s}<-1$ (since $\Gamma_{\omega}^{e} \simeq 0$, $\Gamma_{\omega}^{p h}<0$ and $\nu^{*}$ is large) signaling the instability of the Fermi liquid. This criterion, instead, is not violated in the low-doping limit of the infinite- $U$ three-band Hubbard model (at least in the more physical case with a finite oxygen-oxygen hybridization). This indicates that, even when the antiadiabatic conditions are realized (close to the insulating phase), the large repulsion is able to stabilize the system against bipolaron formation: if polarons are formed, the low frequency analysis indicates that they are stable against bipolaron formation. Of course this does not exclude the possibility af a dynamical binding of the polarons.

A clear confirmation of our results concerning the presence of a lattice-driven instability comes from the numerical exact diagonalization of the three-band Hubbard model with e-ph coupling in a small cluster [53]. This calculations show that a CDW occurs at finite doping (one hole in a cluster of four $\mathrm{Cu}$ atoms with surrounding oxygen) if the e-ph coupling does not exceeds a critical value. Above this value the system enters, instead, a small-polaron regime. It is quite appealing to associate the CDW in the small cluster to the unstable region in the infinite system considered in this paper. Of course, in the case investigated in the present paper by continuously increasing the doping, the instability first occurs at zero wavevectors, but this behaviour could hardly be detected in a finite small cluster, where 
there are few available momenta and where the doping cannot be varied smoothly (the addition of a single hole already produces a doping around 0.25).

Acknowledgements - The authors acknowledge interesting discussions with Prof. C. Di Castro and Dr. R. Raimondi. The authors were supported by the European Economic Community under contract no. SC1* 0222-C(EDB). 


\section{REFERENCES}

[1] See, e.g., Proceedings of the Conference Lattice effects in $H T_{c} S C$, 13-15 January 1991, Santa Fe, USA.

[2] J. P. Carbotte, Rev. Mod. Phys. 62, 10027 (1990).

[3] J. Ranninger, Z. Phys. B 84, 167 (1991) and in Ref. [1]

[4] J. P. Falck, et al. Phys. Rev. Lett. 69, 1109 (1992); P. Calvani, M. Capizzi, S. Lupi, P. Maselli, A. Paolone, P. Roy, S-W. Cheong, W. Sadowski and E. Walker, unpublished.

[5] A. A. Abrikosov, L. P. Gorkov and I. E. Dzyaloshinski, Methods of Quantum Field Theory in Statistical Physics, Ed. Dover, New York.

[6] M. Marder, N. Papanicolau and G. C. Psaltakis, Phys. Rev. B 41, 6920 (1990).

[7] V. J. Emery, S. A. Kivelson, and H. Q. Lin, Phys. Rev. Lett. 64, 475 (1990).

[8] M. Grilli, and G. Kotliar, Phys. Rev. Lett. 64, 1170 (1990).

[9] M. Grilli, C. Castellani, and G. Kotliar, Phys. Rev. B 45, 10805 (1992).

[10] C. Castellani, M. Grilli, and G. Kotliar, Phys. Rev. B 43, 8000 (1991).

[11] N. Cancrini, S. Caprara, C. Castellani, C. Di Castro, M. Grilli and R. Raimondi, Europhys. Lett. 14, 597 (1991).

[12] E. Dagotto, A. Moreo, F. Ortolani, D. Poilblanc and J. Riera, Phys. Rev. B 45, 10741 (1992)

[13] M. Grilli, R. Raimondi, C. Castellani, C. Di Castro, and G. Kotliar, Phys. Rev. Lett. 67, 259 (1991).

[14] M. Grilli, R. Raimondi, C. Castellani, C. Di Castro, and G. Kotliar, International Journal of Modern Physics B Vol. 5, 309 (1991).

[15] R. Raimondi, C. Castellani, M. Grilli, Y. Bang, and G. Kotliar, Phys. Rev. B 47, 3331 (1993).

[16] T. Holstein, Ann. Phys. 8, 325 (1959).

[17] Ju. H. Kim, K. Levin, R. Wentzcovitch, A. Auerbach, Phys. Rev. B 40, 11378 (1989); Ju. H. Kim, K. Levin, R. Wentzcovitch, A. Auerbach, Phys. Rev. B 44, 5148 (1991).

[18] M. Kulić, and R. Zeyher, unpublished.

[19] We recall that the PS is signalled by the occurrence of a diverging compressibility enclosing a region of densities with negative compressibility to be eliminated by a Maxwell construction.

[20] V. J. Emery and S. Kivelson, unpublished; U. Löw, Workshop on "Phase Separation in Cuprate Superconductors", Cottbus, Germany, September 1993.

[21] M. C. Gutzwiller, Phys. Rev. 137, A1726 (1963).

[22] W. F. Brinkman and T. M. Rice, Phys. Rev. B 2, 4302 (1970).

[23] see e.g. P. Nozières, Theory of interacting Fermi systems, Ed. W. A. Benjamin, 1964.

[24] This case (which differs from the intersite e-ph coupling considered in Ref. [17]) will allow us to draw general conclusions using simple Ward identities, Eqs.(11) and (2) below. This general conclusion is easily extended to acustic phonons as well, while the extension of our Ward identity analysis to include intersite e-ph coupling is presently in progress.

[25] These conditions are not very restrictive and are realized, e.g., in the important case of strongly interacting fermions close to a metal-Mott-Hubbard or Charge-Transfer insulator transition.

[26] M. Grilli, G. Kotliar, and A. J. Millis, Phys. Rev. B 42, 329 (1990).

[27] Notice that, within this model, the e-ph couplings $G_{p}$ and $G_{d}$ do not coincide with the 
e-ph coupling $\gamma$ introduced in Section II. In the particular case when $G_{d}=G_{p}=g$ the correspondence is given by the relation $\gamma^{2}=g^{2} / \omega_{0}$.

[28] A more realistic evaluation of the e-ph coupling in cuprates using a screened ionic model can be found in R. Zeyher, Z. Phys. B 80, 187 (1990).

[29] S. E. Barnes, J. Phys. F 6, 1375 (1976).

[30] P. Coleman, Phys. Rev. B 29, 3035 (1984).

[31] N. Read, and D. M. Newns, J. Phys. C 16, 3273 (1983); N. Read, J. Phys. C 18, 2651 (1985).

[32] A. J. Millis, and P. A. Lee, Phys. Rev. B 35, 3394 (1987); G. Kotliar and A. R. Ruckenstein, Phys.Rev. Lett. 57, (1986) 1362.

[33] G. Kotliar, P. A. Lee, and N. Read, Physica C 153-155, 538 (1988).

[34] A different possible approach could also be viable, where the phonons are coupled with the density and not with its fluctuations only. Then the ion equilibrium positions would depend on the tight-binding parameters as well as the doping and should be selfconsistently determined. This latter scheme should be adopted to apply the Maxwell construction needed to determine the PS region, from the analysis of the chemical potential $\mu$ versus the filling $n$. Our simplifying approach assumes that the atomic level energies already include the shift due to the non-zero average of the phonon field. We checked that the two approaches do not give very different results even from the quantitative point of view.

[35] The infinite quasiparticle mass is an outcome of the large- $N$ analysis. At higher order one expects the narrowing of the bandwidth to be limited by the copper superexchange $\mathrm{J}$ [26], [36]. In the cuprates this could limit the mass enhancement to $m^{*} / m \simeq 1.5 t / J \simeq$ $6-8$ [36] in the small doping limit. A larger mass enhancement could be expected in systems with smaller $\mathrm{J} / \mathrm{t}$. A large ratio $\mathrm{m}^{*} / \mathrm{m}$ is also obtained in heavy-fermion systems. In the following the limit $m^{*} / m \rightarrow \infty$ should always be intended with the above provisions.

[36] W. Stephan, and P. Horsch, Phys. Rev. Lett. 66, 2258 (1991).

[37] C. Castellani, G. Kotliar, R. Raimondi, M. Grilli, Z. Wang, and M. Rozenberg, Phys. Rev. Lett. 69, 2009 (1992).

[38] In this $q=0$ and $\omega \rightarrow 0$ limit, the second attractive term in $\Gamma_{\omega}$ also beares a strict resemblance with the corresponding attractive term arising from CT fluctuations in the model of Ref. [15].

[39] The static limit is associated to a differentiation of $G^{-1}$ at fixed chemical potential. Taking the total derivative of $E_{k}$ one has to differentiate with respect to the self-consistency parameters, which, in turn, depend on $\varepsilon_{\alpha}^{0}$ via the self-consistency equations. These latter equations contain Fermi functions which have an implicit dependence on $\varepsilon_{\alpha}^{0}$ via the selfconsistency parameters entering $E_{k}$. Thus, working at fixed chemical potential amounts to consider the (total) derivative of the Fermi functions with respect to $E_{k}$. On the contrary, this derivative should not be considered while performing the calculation in the dynamical limit, which correspond to the fixed doping case.

[40] Our result is in agreement with the one obtained in Ref. [18] for the single-band infinite$\mathrm{U}$ Hubbard model. However, in that case the effect of the static vertex at low doping is much less dramatic since this vertex is proportional to $\left(1+2|\tilde{\mu}| \nu^{*}\right)^{-1}$, where $\tilde{\mu}=$ $-2 t\left(\cos \left(k_{F x}\right)+\cos \left(k_{F y}\right)\right)$ vanishes when $\delta \rightarrow 0$. This latter effect simply arises from 
the specific form of the Fermi surfaces in the single-band Hubbard model, where the constant energy surfaces are determined by the condition $\cos \left(k_{x}\right)+\cos \left(k_{y}\right)=$ constant. Then, at $\delta=0$, the Fermi energy reduces to a square where the constant is zero. This peculiarity of the single-band Hubbard model is not present once next nearest neighbor hopping terms are considered, which modify the shape of the Fermi surface. In this latter case $\tilde{\mu}$ no longer vanishes at half-filling and the presence of a large quasiparticle density of states $\nu^{*}$ in the denominator should lead to a large suppression of the e-ph interaction.

[41] We notice, for instance, that the attractive $\lambda_{s}$ at $\delta=0.208$ in Table 1 is still rather small despite the closeness to the instability $\left(\delta_{c}=0.210\right)$, where $\lambda_{s}$ is expected to diverge. This shows that to get a large $\lambda_{s}$ requires a quite fine tuning of the model parameters.

[42] J. P. Falck, A. Levy, M. A. Kastner, and R. J. Birgenau, Phys. Rev. Lett. 69, 1109 (1992).

[43] F. W. de Wette, A. D. Kulkarni, Phys. Rev. B 46, 14922 (1992).

[44] H. Nozaki, and S. Itoh, Phys. Rev. B 48, 7583 (1993).

[45] H. Krakauer, W. E. Pickett, and R. E. Cohen, Phys. Rev. B 47, 1002 (1993).

[46] Notice that, according to the analysis of Ref. [17], the reduction of the e-ph vertex, which we obtain in the small frequency limit, also occurs for the covalent e-ph coupling. In this latter case the e-ph coupling, being proportional to the hopping $t_{p d}$, is reduced by a factor $r_{0}^{2}$ already at mean field level; however, a detailed dynamical analysis of this reduction is still lacking.

[47] A. B. Migdal, Sov. Phys. JETP 34, 996 (1958).

[48] L. Pietronero and S. Strässler, Europhys. Lett. 18 (7), 627 (1992)

[49] In the case of optical phonons, which contribute negligibly to the quasiparticle scattering in the zero temperature limit, the depression will concern their contribution at finite temperature.

[50] H. Rietschel and L. J. Sham, Phys. Rev. B 28, 5100 (1983); M. Grabowski and L. J. Sham, Phys. Rev. B 29, 6132 (1984).

[51] J. H. Kim and Z. Tesanovic, Phys. Rev. Lett. 71, 4218 (1993).

[52] H. De Raedt, and Ad. Lagendijk, Phys. Rev. B 27, 6097 (1983); Phys. Rev. B 30,1671 (1984).

[53] A. Dobry, A. Greco, J. Lorenzana, and J. Riera, unpublished. 


\section{FIGURE CAPTIONS}

FIG. 1: Phase diagram $\left(\varepsilon_{p}^{0}-\varepsilon_{d}^{0}\right) / t_{p d}$ versus positive doping $\delta$ for $t_{p p}=0, G_{p}=0.15 t_{p d}$, $G_{d}=0.1 t_{p d}$, and $\omega_{0}=0.02 t_{p d}$.

FIG. 2: Phase diagram $\left(\varepsilon_{p}^{0}-\varepsilon_{d}^{0}\right) / t_{p d}$ versus positive doping $\delta$ for $t_{p p}=0.2 t_{p d}, G_{p}=0.15 t_{p d}$, $G_{d}=0.1 t_{p d}$, and $\omega_{0}=0.02 t_{p d}$.

FIG. 3: Effective static quasiparticle scattering amplitude versus transferred momentum well outside $(\delta=0.2$, dotted line), slightly outside $(\delta=0.225$, continuous line) and well inside $(\delta=0.3$, dashed line) the unstable region. The parameter are as in Fig. 2 with $\varepsilon_{p}^{0}-\varepsilon_{d}^{0}=4.9 t_{p d}$. The instability occurs at $\delta_{c}=0.23$. The scattered quasiparticles are on the Fermi surface and $q$ is in the $y$ direction, $\mathbf{q}=(0, q)$.

FIG. 4: a) Diagrammatic representation of the renormalized quasiparticle-phonon vertex. The electronically screened vertex is reported in b), where the open dot is the bare phonon-quasiparticle vertex and the thick dashed line is the resummed slave-boson propagator diagrammatically represented in c). No phonon propagators are included in the resummation c).

FIG. 5: Interaction-renormalized quasiparticle-phonon vertex at fixed low $(q=0.06$, continuous line) and large momenta $(q=2.20$, dashed line) as a function of the transferred Matsubara frequency $\omega$. The parameters are as in Fig.3 with $\delta=0.225$. The scattered quasiparticles are on the Fermi surface and $q$ is in the $y$ direction, $\mathbf{q}=(0, q)$. The inset shows an enlargement of the low frequency region. The quasiparticle density of states is $\nu^{*} \simeq 13 / t_{p d}$ per cell. The vertical lines indicate the phonon frequency and half the quasiparticle bandwidth.

FIG. 6: Effective dynamic full-phonon-mediated scattering amplitude at fixed low ( $q=0.06$, continuous line $)$ and large momenta $(q=2.20$, dashed line) versus transferred Matsubara frequency. The parameters are as in Fig.3. The calculations are performed well outside ( $\delta=0.2$, diamonds) and slightly outside $(\delta=0.225$, crosses $)$ the unstable region. The scattered quasiparticles are on the Fermi surface and $q$ is in the $y$ direction, $\mathbf{q}=(0, q)$. The quasiparticle density of states is $\nu^{*} \simeq 13 / t_{p d}$ per cell.

FIG. 7: (a) Effective dynamic quasiparticle total (continuous line), purely electronic repulsive (dashed line) and full-phonon (dotted line) scattering amplitudes in the Cooper channel at fixed low momentum $(q=0.06)$ as a function of the transferred Matsubara frequency. The parameters are as in Fig.3. The calculations are performed slightly outside $(\delta=0.225)$ the unstable region. The scattered quasiparticles are on the Fermi surface and $q$ is in the $y$ direction, $\mathbf{q}=(0, q)$. The quasiparticle density of states is $\nu^{*} \simeq 13 / t_{p d}$ per cell. The vertical lines indicate the phonon frequency and half the quasiparticle bandwidth. (b) Same as in (a) but at large momentum $(q=2.20)$.

FIG. 8: (a) Effective dynamic quasiparticle total (continuous line), purely electronic repulsive (dashed line) and full-phonon (dotted line) scattering amplitudes in the Cooper channel at fixed low $(q=0.07)$ versus transferred Matsubara frequency. The calculations are performed at $\delta=0.18$, close to the unstable region, occurring at $\delta_{c}=0.185$. The phonon frequency is $\omega=0.08 t_{p d}, G_{p}=0.3 t_{p d}$ and $G_{d}=0.1 t_{p d}$. The scattered quasiparticles are on the Fermi surface and $\mathbf{q}=(0.053,0.03)(|q|=0.06)$. The quasiparticle density of states is $\nu^{*} \simeq 9 / t_{p d}$ per cell. The vertical lines indicate the phonon frequency and half the quasiparticle bandwidth. (b) Same as in (a) but with large momenta $\mathbf{q}=(0,2.25)$.

FIG. 9: Effective dynamic quasiparticle total (continuous line), purely electronic repul- 
sive (dashed line) and full-phonon (dotted line) scattering amplitudes in the Cooper channel at fixed low momentum $(q=0.083)$ versus transferred Matsubara frequency. The phonon frequency is $\omega=0.02 t_{p d}, G_{p}=0.15 t_{p d}$ and $G_{d}=0.1 t_{p d}$ and the calculations are performed at $\delta=0.2$, far from the unstable region, occurring at $\delta_{c}=0.225$. The scattered quasiparticles are on the Fermi surface and $\mathbf{q}=(0.078,0.028)(|q|=0.083)$. The quasiparticle density of states is $\nu^{*} \simeq 9 / t_{p d}$ per cell. The vertical lines indicate the phonon frequency and half the quasiparticle bandwidth. 


\section{TABLE CAPTIONS}

TABLE 1: $s_{1}$ - and $d_{1}$-wave coupling constants for various doping and for the model with $\varepsilon_{p}^{0}-\varepsilon_{d}^{0}=3.3 t_{p d}, t_{p p}=0, G_{p}=0.15 t_{p d}, G_{d}=0.1 t_{p d}$, and $\omega_{0}=0.02 t_{p d}$. The instability line is at $\delta_{c}=0.21$.

TABLE 2: $s_{1^{-}}, s_{2^{-}}, d_{1^{-}}$and $d_{2^{-}}$wave coupling constants for various doping and for the model with $\varepsilon_{p}^{0}-\varepsilon_{d}^{0}=4.9 t_{p d}, t_{p p}=0.2 t_{p d}, G_{p}=0.15 t_{p d}, G_{d}=0.1 t_{p d}$, and $\omega_{0}=0.02 t_{p d}$. The instability line is at $\delta_{c}=0.23$. 


\section{TABLES}

TABLE 1

\begin{tabular}{|c|r|r|r|r|}
\hline \hline \hline$\delta$ & 0.15 & 0.20 & 0.208 & 0.22 \\
\hline$\lambda_{s_{1}}$ & -0.75 & -0.5 & 0.43 & -0.45 \\
$\lambda_{d_{1}}$ & -0.044 & 0.2 & 0.35 & \\
\hline \hline
\end{tabular}

TABLE 2

\begin{tabular}{|c|r|r|c|}
\hline \hline$\delta$ & 0.15 & 0.20 & 0.229 \\
\hline$\lambda_{s_{1}}$ & -0.58 & -0.48 & 1.2 \\
$\lambda_{s_{2}}$ & -0.55 & -0.65 & 1.5 \\
$\lambda_{d_{1}}$ & -0.063 & 0.017 & 2.1 \\
$\lambda_{d_{2}}$ & -0.021 & 0.012 & 1.8 \\
\hline \hline
\end{tabular}

